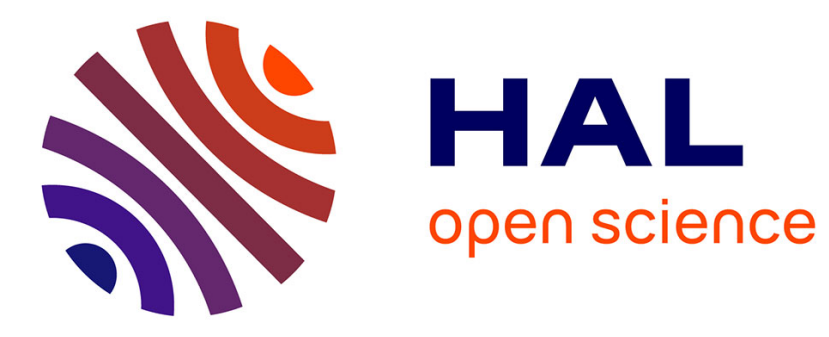

\title{
Wave turbulence in vibrating plates
}

Olivier Cadot, M Ducceschi, Thomas Humbert, Benjamin Miquel, N Mordant, C Josserand, Cyril Touzé

\section{To cite this version:}

Olivier Cadot, M Ducceschi, Thomas Humbert, Benjamin Miquel, N Mordant, et al.. Wave turbulence in vibrating plates. Christos H. Skiadas, Charilaos Skiadas. Handbook of applications of chaos theory, Chapman and Hall/CRC, 2016, 9781466590434. hal-01325042

\section{HAL Id: hal-01325042 \\ https://hal-ensta-paris.archives-ouvertes.fr/hal-01325042}

Submitted on 1 Jun 2016

HAL is a multi-disciplinary open access archive for the deposit and dissemination of scientific research documents, whether they are published or not. The documents may come from teaching and research institutions in France or abroad, or from public or private research centers.
L'archive ouverte pluridisciplinaire HAL, est destinée au dépôt et à la diffusion de documents scientifiques de niveau recherche, publiés ou non, émanant des établissements d'enseignement et de recherche français ou étrangers, des laboratoires publics ou privés. 
1

\section{Wave turbulence in vibrating plates}

O. Cadot

M. Ducceschi

T. Humbert

B. Miquel

N. Mordant

C. Josserand

C. Touzé
1.1 Introduction $\ldots \ldots \ldots \ldots \ldots \ldots \ldots \ldots \ldots \ldots \ldots \ldots \ldots, \mathbf{1}-1$

1.2 Theoretical results $\ldots \ldots \ldots \ldots \ldots \ldots \ldots \ldots \ldots \ldots \ldots, 1-3$

Nonlinear vibrations of plates: von Karman model •

Wave turbulence theory for vibrating plates •

Nonstationary wave turbulence

1.3 Numerical results .........................

Kolmogorv-Zakharov spectrum • Nonstationary wave

turbulence $\bullet$ Effect of an imperfection

1.4 Real plates .............................. $1-12$

First experimental results $\bullet$ Weak nonlinearity and

separation of timescales $\bullet$ Finite-size effects $\bullet$ Effect of damping on the slope of the power spectra $\bullet$

Dependence on injected power

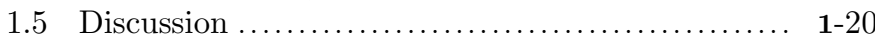

1.6 Conclusion $\ldots \ldots \ldots \ldots \ldots \ldots \ldots \ldots \ldots \ldots \ldots \ldots \ldots \ldots, 1-22$

References ................................... 1-24

\section{$1.1 \quad$ Introduction}

Turbulence is a general term used for describing the erratic motions displayed by nonlinear systems that are driven far from their equilibrium position and thus display complicated motions involving different time and length scales. Without other precision, the term generally refers to hydrodynamic turbulence, as the main field of research has been directed towards irregular motions of fluids and the solutions of Navier-Stokes equations. During the $\mathrm{XX}^{\text {th }}$ century, the theory has shown important breakthrough thanks to the qualitative ideas of Richardson and the quantitative arguments of Kolmogorov that culminated in the so-called K41 theory [Kol41a, Kol41b, Fri95]. This statistical approach, although giving successful predictions, still faces an irreducible obstacle due to the lack of closure in the infinite hierarchy of moment equations.

Wave turbulence (WT) share many common ideas with turbulence, in particular as being a statistical theory for out-of-equilibrium systems. A main difference resides in the fact that the persistence of waves is assumed. By considering a sea of weakly interacting dispersive wave trains, it has been shown that a natural asymptotic closure may be derived from the hierarchy of moment equations [BN67, ZLF92, NNB01]. Furthermore, this closure equation, referred to as the kinetic equation, has been shown to admit two sets of stationary solutions [ZF67a, ZF67b, ZLF92]. The first one is the classical equipartition of energy. Most importantly, the second one describes an energy flux through the scales and thus recovers the Richardson's picture of turbulence with a cascade of energy from the injection to the dissipative scales. Inbetween, an inertial range with a conservative Hamiltonian dynamics is assumed. 
A salient feature of the wave turbulence (WT) theory is that solutions of the kinetic equation are analytic, hence yielding accurate predictions for the stationary repartition of energy through scales in a given out-of-equilibrium system. The solutions of the kinetic equation have been derived starting from the mid-1960s and correspond to energy spectra with power-law dependence on the wavenumber. The theory has been successfully applied to capillary [ZF67b, PZ96] or gravity [ZF67a, $\left.\mathrm{OOS}^{+} 02\right]$ waves on the surface of liquids, to plasmas [MRZ95], to nonlinear optics [DNPZ92], to magnetohydrodynamics [NNG01] or even to Bose-Einstein condensates [LNW03]. The theoretical bases are now firmly established and the reader is referred to the existing books [ZLF92, Naz11] or review paper [NR11] for a complete picture of the existing literature.

The application of WT to vibrating plates started with the theoretical derivation of the kinetic equation from the dynamical von Kármán equations [vK10, CH56, LL59] that describe large-amplitude motions of thin plates [DJR06]. Since this date, numerous papers have been published covering experimental, theoretical and numerical materials. In fact, it appears that the vibrating plate is a perfect candidate for a thorough comparison of experiments with theoretical predictions. As compared to other physical systems such as capillary or gravity waves for example, an experimental set-up with a fine control of energy injection and a confortable range of wavelength is not too difficult to put in place. Secondly, the available measurement techniques allow one to get a complete and precise picture of the dynamics through the scales, both in the space and frequency domains. Finally, numerical codes with good accuracy have been developed so that all the underlying assumptions of the theory as well as its predictions have been tested, both on the experimental and the numerical levels.

The first experimental papers reported a discrepancy between the theoretical predictions and the measurements [BCOT08, Mor08]. An important research effort has then been undertaken in order to understand the origin of these differences. The aim of the present paper is to sum up the most important results obtained so as to give an overview of the solved problems and open issues. The paper is organized as follows:

- The first section is devoted to theoretical results. It starts with a description of the von Kármán equations for geometrically nonlinear vibrations of plates, and recalls the main assumptions underlying the mechanical model. The application of the wave turbulence theory to the von Kármán model is then overviewed. Stationary solutions of the kinetic equation are given, as well as self-similar laws for non-stationary turbulence.

- Section 1.3 sums up the numerical results obtained for stationary and nonstationary turbulence, including the effect of a simple imperfection.

- Section 1.4 gathers the experimental confrontations to the theory, and reviews all the underlying assumptions of wave turbulence and their experimental verifications, in order to explain the origin of the discrepancies first reported.

- A general discussion is given and the conclusions are drawn. 


\subsection{Theoretical results}

\subsubsection{Nonlinear vibrations of plates: von Kármán model}

Mechanical models for large-amplitude, geometrically nonlinear vibrations of thin plates are numerous and rely on a set of assumptions and approximations that give the level of needed accuracy, depending on the vibratory state, the thickness and the frequency range. The von Kármán model is one of the simplest one and relies on strong assumptions that may be violated, especially when the plate is not too thin, or when the frequency range of the vibrations include high-frequency components. It is largely used as it writes as a simple set of coupled nonlinear partial differential equations that are amenable to analytical approaches [LL59, NM79, BC91, SMN75, TTC02]. Moreover, it is known to produce very accurate results for very thin plates and for vibration amplitudes up to one to five times the thickness [YK05, TBC12, TVC13, TVC14].

As a description of geometrically nonlinear vibrations, the material is assumed to be linear elastic. Isotropy is also assumed for simplicity here, so that the material is fully described thanks to its Young modulus $E$, Poisson ratio $\nu$ and density $\rho$. The main assumptions for deriving the von Kármán model are the following [vK10, CH56, LL59, NM79, TB08]:

- The Kirchhoff-Love kinematical assumptions are fulfilled. This implies in particular that the transverse shear stresses are neglected. Rotation angles are assumed to be small so that the displacement of any point of the mid-surface of the plate is parameterized with the three displacements $(u, v, w)$ only.

- The normal stress along the transverse direction is neglected.

- A particular truncation in the longitudinal part of the Green-Lagrange strain tensor, due to von Kármán [vK10, Cia80, MHC97], is used.

- Rotatory inertia is neglected.

With these assumptions a von Kármán model with the three displacements $(u, v, w)$ is obtained, see e.g. [KY04, YK05, BTTD14]. A last assumption consists in neglecting the in-plane inertia. In this case an Airy stress function $F$ can be introduced and the two longitudinal displacements $(u, v)$ can be condensated, as proposed by Föppl [Föp07, BC91]. This model, generally referred to as Föppl - von Kármán, depends only on the two unknowns $w(\mathbf{x}, t)$ and $F(\mathbf{x}, t)$ (where $\mathbf{x}$ is the two-dimensional space position and $t$ the time), and reads, for an undamped perfect plate without external forcing:

$$
\begin{aligned}
\rho h \ddot{w}+D \Delta \Delta w & =L(w, F), \\
\Delta \Delta F & =-\frac{E h}{2} L(w, w) .
\end{aligned}
$$

In these equations: $h$ is the thickness, $D=E h^{3} / 12\left(1-\nu^{2}\right)$ the bending rigidity, $\Delta$ the laplacian and $L$ a bilinear differential operator which reads, in cartesian coordinates: $L(f, g)=f_{, x x} g_{, y y}+f_{, y y} g_{, x x}-2 f_{, x y} g_{, x y}$.

\subsubsection{Wave turbulence theory for vibrating plates}

This section is devoted to the application of the Wave turbulence (WT) theory to the von Kármán equations for large-amplitude vibrations of thin plates. The underlying assumptions needed for deriving the theory are the following:

1. The linearized system is composed of dispersive waves.

2. Weak nonlinearity is assumed, so that the nonlinear terms can be ordered and considered small as compared to the linear ones. 
3. A clear separation of time scales exists between the linear oscillations and the nonlinear time upon which energy is nonlinearly exchanges between wavetrains.

4. An inertial range with a conservative dynamics exists.

5. The system is of infinite size.

Assumptions 2 and 3 are correlated and state that the dynamics is composed of a sea of weakly interacting, persistent waves. Formally speaking, this framework should thus be referred to as a "weak, wave turbulence theory". However for the sake of simplicity we will name it under the general term "wave turbulence" (WT) in the remainder of the paper. Thanks to the second assumption, one can derive a hierarchy of equations for the different moments of the field. An equation for the wave spectrum is eventually deduced using long time asymptotics. This kinetic equation can be obtained equivalently using slightly different arguments: the so-called random phase approximation [ZLF92, Naz11] or an asymptotic expansion for the cumulants [NR11].

More precisely, the von Kármán (VK) equations are first written in Fourier space by introducing the transforms: $W_{\mathbf{k}}(t)=\frac{1}{2 \pi} \int w(\mathbf{x}, t) \mathrm{e}^{i \mathbf{k x}} \mathrm{d}^{2} \mathbf{x}$ and $F_{\mathbf{k}}(t)=\frac{1}{2 \pi} \int F(\mathbf{x}, t) \mathrm{e}^{i \mathbf{k x}} \mathrm{d}^{2} \mathbf{x}$ for the two unknown fields, yielding:

$$
\begin{aligned}
& \rho h \ddot{W}_{\mathbf{k}}=-D k^{4} W_{\mathbf{k}}+L_{\mathbf{k}}(w, F), \\
& k^{4} F_{\mathbf{k}}=-\frac{E h}{2} L_{\mathbf{k}}(w, w),
\end{aligned}
$$

where $L_{\mathbf{k}}$ denotes the Fourier transform of the bilinear operator $L$. From the linear terms, the dispersion relation between the wavenumber $\mathbf{k}$ and the radian frequency of the wave $\omega_{k}$ is retrieved as:

$$
\omega_{k}=\sqrt{\frac{E h^{2}}{12 \rho\left(1-\nu^{2}\right)}} k^{2} .
$$

In order to write the VK equations under its canonical Hamiltonian form, one introduces the momentum $Y_{\mathbf{k}}=\rho \partial_{t} W_{\mathbf{k}}$, as well as the canonical variables $A_{\mathbf{k}}$ defined by: $W_{\mathbf{k}}=\frac{X_{k}}{\sqrt{2}}\left(A_{\mathbf{k}}+A_{-\mathbf{k}}^{\star}\right)$ and $Y_{\mathbf{k}}=\frac{-i}{\sqrt{2 X_{k}}}\left(A_{\mathbf{k}}-A_{-\mathbf{k}}^{\star}\right)$ with $X_{k}=1 / \sqrt{\rho \omega_{k}}$. This results in a formulation with a diagonalized linear part where the nonlinear term appears clearly as a perturbation to the linear wave equation for small wave amplitudes. Finally, separating the dynamics within the linear time scale of the waves and the long time nonlinear interactions allows us to write: $A_{\mathbf{k}}=a_{\mathbf{k}} \mathrm{e}^{i \omega_{k} t}$, where the variation of the amplitude $a_{\mathbf{k}}$ is slow as compared to the oscillation time $1 / \omega_{k}$. Formally, the linear dynamics of the modes can be suppressed in this slow time scale analysis so that we end up with a kinetic equation for the second order moment defined by $<a_{\mathbf{k}_{1}} a_{\mathbf{k}_{2}}^{\star}>=n_{\mathbf{k}_{1}} \delta^{(2)}\left(\mathbf{k}_{1}+\mathbf{k}_{2}\right)$, which reads for $n_{\mathbf{k}} \equiv n(\mathbf{k}, t)$ :

$$
\frac{\partial n_{\mathbf{k}}}{\partial t}=I(\mathbf{k}) \text {. }
$$

The expression of the collision integral $I(\mathbf{k})$ comes from the nonlinear interaction between four waves (see [DJR06] for more details), and reads:

$$
\begin{aligned}
I(\mathbf{k})=12 \pi \int \mid & \left.J_{k 123}\right|^{2} f_{k 123} \delta\left(\mathbf{k}+s_{1} \mathbf{k}_{1}+s_{2} \mathbf{k}_{2}+s_{3} \mathbf{k}_{3}\right) \\
& \times \delta\left(\omega_{k}+s_{1} \omega_{1}+s_{2} \omega_{2}+s_{3} \omega_{3}\right) \mathrm{d} \mathbf{k}_{1} \mathrm{~d}_{2} \mathrm{dk}_{3},
\end{aligned}
$$

where $J_{k 123}$ stands for the interaction term itself and $f_{k 123}$ is such that

$$
f_{k 123}=\sum_{s_{1}, s_{2}, s_{3}} n_{\mathbf{k}} n_{\mathbf{k}_{1}} n_{\mathbf{k}_{2}} n_{\mathbf{k}_{3}}\left(\frac{1}{n_{\mathbf{k}}}+\frac{s_{1}}{n_{\mathbf{k}_{1}}}+\frac{s_{2}}{n_{\mathbf{k}_{2}}}+\frac{s_{3}}{n_{\mathbf{k}_{3}}}\right) .
$$


Here, the notation $s_{i}$ stands for $s_{i}= \pm 1$ so that the collision integral corresponds to four waves interaction, with both $2 \leftrightarrow 2$ and $3 \leftrightarrow 1$ waves mechanisms [Naz11, DJR06]. As it has been shown for water waves, two distinct types of stationary solutions exist in general for such kinetic equation. First, the equipartition of energy between the modes, since $n_{k} \propto 1 / \omega_{k}$ is a trivial root of the integral term (1.5). This is the so-called Rayleigh-Jeans (RJ) spectrum, and it writes in Fourier space:

$$
n_{\mathbf{k}} \propto \frac{1}{k^{2}} \text { giving for the Fourier spectrum of the displacement } \quad<\left|W_{\mathbf{k}}\right|^{2}>\propto \frac{1}{k^{4}} .
$$

In addition, another solution can be exhibited that exactly vanishes the full integral as first shown for water waves by Zakharov [ZF67a, ZF67b] and therefore called KolmogorovZakharov (KZ) spectrum. It involves a constant flux of energy $\epsilon$ that is transferred from the large scales (formally $k=0$ in the mathematical solution) to the small scales (mathematically $k \rightarrow \infty$ and practically towards a scale where dissipation becomes dominant). This solution including a cascade of energy to the small scales reads:

$$
n_{k}^{K Z}=C \frac{h \varepsilon^{1 / 3} \rho^{2 / 3}}{\left(12\left(1-\nu^{2}\right)\right)^{2 / 3}} \frac{\ln ^{1 / 3}\left(k_{\star} / k\right)}{k^{2}} \text { or }<\left|W_{\mathbf{k}}\right|^{2}>\propto \frac{\ln ^{1 / 3}\left(k_{\star} / k\right)}{k^{4}} .
$$

This solution has a particular structure compared to the usual power-laws: indeed, this solution is the same than the RJ spectrum but a logarithm correction. This peculiar structure, similar to the one observed for the nonlinear Schrödinger equation [DNPZ92], comes from the degeneracy of the KZ solution so that the next order term in the collision integral has to be considered (see [DJR06, Dür10, DJR] for more details). This logarithmic correction involves a cut-off wave number $k_{\star}$ above which the mathematical solution is no more valid. The mathematical function vanishes at $k=k_{\star}$ so that everything works as if the constant flux $\epsilon$ would be absorbed at $k=k_{\star}$. In fact, since the logarithmic correction is obtained as a second order expansion, it is valid only for $k \ll k_{\star}$, so that one expects practically that the spectrum simply decreases rapidly around and above $k_{\star}$. Such KZ solution can be observed when energy is injected in the system at large scale (small $k$ ) and dissipated at small scale (large $k$ ) so that one observes a transition between the inertial regime (where the constant flux holds) and the dissipative range.

This KZ spectrum can be written in term of the energy spectrum, as a function of $k$, or as a function of $\omega$ (or $f$ ), following,

$$
P_{v}(\mathbf{k})=\frac{\bar{C} h}{\left(1-\nu^{2}\right)^{2 / 3}} \varepsilon^{1 / 3} \log ^{1 / 3}\left(\frac{k_{\star}}{k}\right) \text { and } P_{v}(f)=\frac{\tilde{C} h}{\left(1-\nu^{2}\right)^{2 / 3}} \varepsilon^{1 / 3} \log ^{1 / 3}\left(\frac{f^{\star}}{f}\right),
$$

where

$$
f^{\star}=\frac{1}{2 \pi} \sqrt{\frac{E h^{2}}{12 \rho\left(1-\nu^{2}\right)}} k_{\star}^{2} .
$$

We notice that the energy spectrum is almost flat since it varies only through the logarithmic correction.

\subsubsection{Nonstationary wave turbulence}

Other properties of the solutions of the kinetic equation can de derived by considering nonstationary evolutions. In this particular case, one is able to exhibit self-similarity laws that must be fulfilled by the solutions [ZLF92, FS91, CNP03, DCTB14]. Let us consider a self-similar solution for the wave spectrum which depends only on the wavevector modulus, 
$n(\mathbf{k}, t)=t^{-q} f\left(k t^{-p}\right)=t^{-q} f(\eta)$. Introducing this expression in the kinetic equation (1.4), and taking into account the expression of $\left|J_{k 123}\right|^{2}$ [DJR06, Dür10, DJR, DCTB14], one obtains the following relationship

$$
-t^{-q-1}\left[q f(\eta)+p \eta f^{\prime}(\eta)\right]=I(\eta) t^{-3 q+2 p},
$$

so that a self-similar solution for the wave spectrum exists if the condition $-q-1=-3 q+2 p$, is satisfied.

Let us now consider two different cases of non-stationary evolution of the wave turbulence spectrum for the system, in order to derive another condition that must be fulfilled by the unknowns $p$ and $q$ :

Case 1, forced turbulence : We assume that the plate is forced by a sinusoidal pointwise forcing of constant amplitude and excitation frequency. In this case the total energy $\xi=\int \omega n_{\mathbf{k}} \mathrm{d} \mathbf{k}$ increases linearly with time so that $\xi \sim t$.

Case 2, free turbulence : The plate is left free to vibrate, given an amount of energy as initial condition. In this case the total energy is constant so that $\xi \sim t^{0}$.

Using the self-similar assumption for the wave vector in the energy equation obtained for the two cases considered, yields the following relationships:

$$
4 p-q= \begin{cases}1 & \text { for the forced turbulence (case 1) } \\ 0 & \text { for the free turbulence (case 2) }\end{cases}
$$

Solving for $(p, q)$ in both cases give

$$
\begin{aligned}
& \text { forced turbulence : } \quad p=1 / 2, \quad q=1 \text {, } \\
& \text { free turbulence : } \quad p=1 / 6, \quad q=2 / 3 \text {. }
\end{aligned}
$$

The self-similar laws obtained for the wave spectrum can be translated for the power spectrum of the transverse velocity $P_{v}(\omega)$, where $v=\dot{w}$. One obtains for forced vibrations [DCTB14]:

$$
P_{v}(\omega, t) \sim f_{1}\left(\sqrt{\frac{\omega}{t}}\right)=g_{1}\left(\frac{\omega}{t}\right)
$$

and for free vibrations (case 2):

$$
P_{v}(\omega, t) \sim t^{-1 / 3} f_{2}\left(\sqrt{\frac{\omega}{t^{1 / 3}}}\right)=t^{-1 / 3} g_{2}\left(\frac{\omega}{t^{1 / 3}}\right),
$$

where $g_{1,2}$ (or $f_{1,2}$ ) have been indexed with respect to case 1 and 2 , and are functions to be defined. The first relationship underlines the fact that, when the plate is excited by an external harmonic forcing of constant amplitude and frequency, the cascade should show a front propagating linearly in frequency with respect to time. More specifically, denoting $\omega_{c}$ the frequency of the front, one should observe that $\omega_{c} \propto t$, and that the amplitude of the power spectrum at the front $P_{v}\left(\omega_{c}\right)$ should stay constant. In the second case where the plate is left free to vibrate, given an initial amount of energy, one should observe the front of the cascade propagating as $t^{1 / 3}$ while the amplitude of the spectrum at the front should decrease as $t^{-1 / 3}$. These two predictions will be confronted to numerical simulations in section 1.3.2. 


\subsection{Numerical results}

\subsubsection{Kolmogorv-Zakharov spectrum}

Numerical simulations of the VK equation can be performed using a pseudo-spectral method that takes advantage of the linear dynamics of the vibrating plate. In that purpose, we use in Fourier space the reduced variable $Z_{\mathbf{k}}=W_{\mathbf{k}} e^{i \omega_{k} t}$ so that the dynamical equation for $Z_{\mathbf{k}}$ involves only the nonlinear terms. This terms are then computed in real space first and then transform in the Fourier space in order to integrate the dynamics using an Adams-Bashford scheme.

In order to test the KZ spectrum, we need to impose to the system conditions that are consistent with the energy cascading from large to small scales. We thus add two terms in the VK equation (1.2) in the Fourier space:

$$
\begin{aligned}
& \rho h \ddot{W}_{\mathbf{k}}=-D k^{4} W_{\mathbf{k}}+L_{\mathbf{k}}(w, F)+I_{\mathbf{k}}+d_{\mathbf{k}} \dot{W}_{\mathbf{k}}, \\
& k^{4} F_{\mathbf{k}}=-\frac{E h}{2} L_{\mathbf{k}}(w, w) .
\end{aligned}
$$

In these equations, the injection term $I_{\mathbf{k}}$ is modeled by a random forcing valid at large scale (for $|k|<k_{i}$ only where $2 \pi / k_{i}$ is the injection scale):

$$
I_{\mathbf{k}} \propto \Theta \text { for }|k|<k_{i} \text { and } I_{\mathbf{k}}=0 \text { elsewhere }
$$

where $\Theta$ is a classical random process. On the other hand, an idealized linear dissipation is used, acting only for $|k|>k_{d}$, where $2 \pi / k_{d}$ is the dissipative or Kolmogorov scale, yielding:

$$
d_{\mathbf{k}} \propto\left(k^{2}-k_{d}^{2}\right) \text { for }|k|>k_{d} \text { and } d_{\mathbf{k}}=0 \text { elsewhere. }
$$

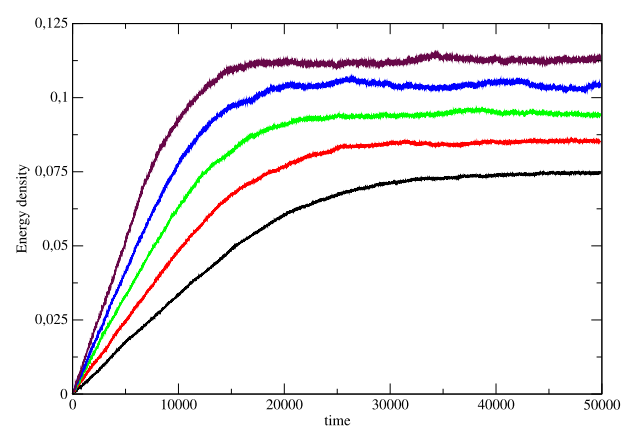

(a)

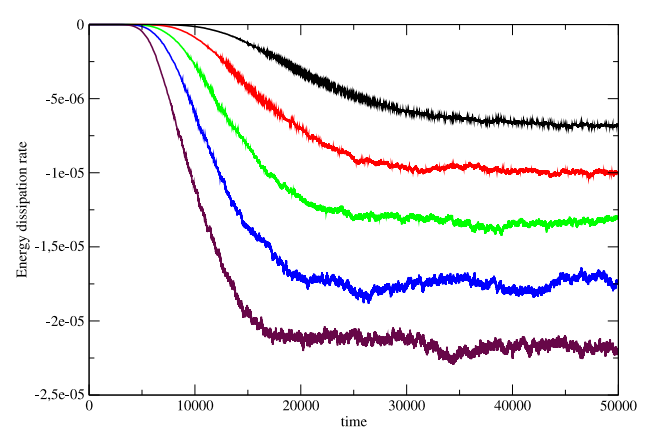

(b)

FIGURE 1.1 a) Evolution of the energy density with time for different forcing amplitudes obtained by solving numerically the dimensionless VK equations. The mesh size is $d x=0.5$ on a $1024 \times 1024$ grid so that the size of the plate is $512 \times 512$ in dimensionless unit $\ell$. The random forcing holds for $|k|<k_{i}=0.1$ while the dissipation acts for $|k|>k_{d}=5.5$. b) The energy dissipation rate as a function of time for the same forcing amplitudes.

We investigate numerically the dynamics of this system by changing only the forcing amplitude, all the other terms remaining the same. Moreover, we use the dimensionless 
version of the VK equations where the lengthes are expressed in unit of $\ell=h / \sqrt{3\left(1-\nu^{2}\right)}$, the times in unit of $\sqrt{\rho / E} \ell$ and the Airy function $F$ in units of $E h \ell^{2}$. Fig. 1.1(a) shows the evolution of the energy in the system for different values of the injection amplitude. After a transient, a stationary regime is reached as illustrated on Fig. 1.1(b) where the dissipated energy rate $\epsilon$ is plotted as function of time. In this steady state, this constant dissipated energy corresponds to the flux of energy that cascades from the large scale toward the small scales.

Finally, Fig. 1.2 shows the energy spectra in the stationary regime for these different forcing amplitudes as functions of the frequency. The energy spectra have been rescaled by $\epsilon^{1 / 3}$, following the theoretical prediction (1.9). We observe that the different spectra collapse in a single curve that is well fitted by the WT solution $\log ^{1 / 3}\left(f_{c}^{\star} / f\right)$. Amazingly, $f_{c}^{\star}$ corresponds exactly to the frequency of the wavenumber $k_{d}$.

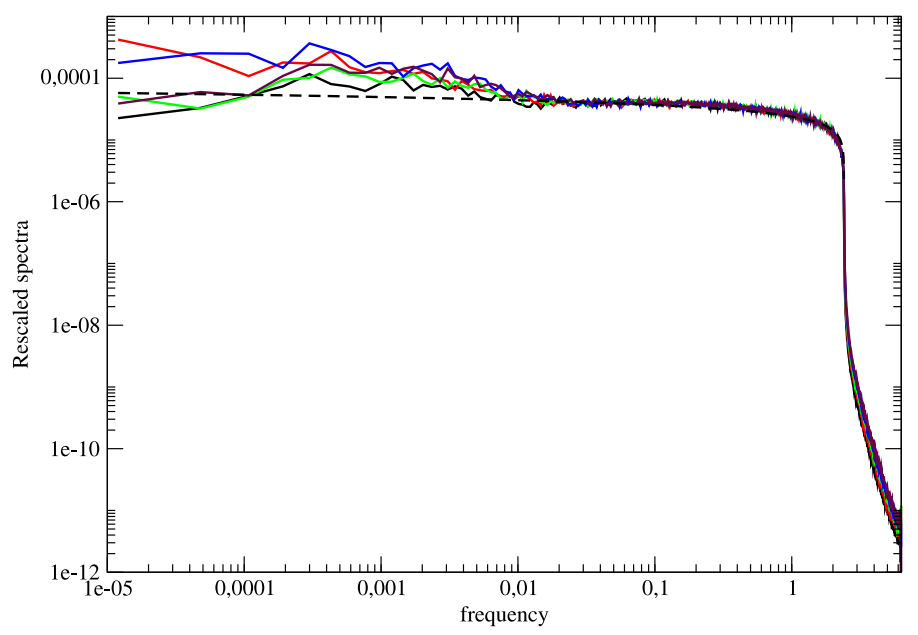

FIGURE 1.2 Rescaled spectra for different forcing amplitudes (same parameters as in Figs. (1.1)). The spectra are rescaled by the dissipation rate $\epsilon^{1 / 3}$ and collapse well into a single curve for $f_{i}<f<f_{c}^{\star}$, where $f_{i}$ is the injection frequency corresponding to the wave number $k_{i}$. The black dotted line shows the fit of this curve by the theoretical law (1.9), exhibiting a good agreement.

\subsubsection{Nonstationary wave turbulence}

This section offers numerical illustrations of the theoretical results for nonstationary turbulence introduced in section 1.2.3. The numerical method chosen here is a finite difference, energy-conserving scheme. As opposed to the pseudo-spectral method used in section 1.3.1, finite difference simulations take place entirely in physical space. A family of such algorithms has been provided by [Bil08a], where discrete energy conservation properties give a strict stability condition and a bound on the solution growth. Such schemes, originally thought for use in sound synthesis [Bil08b], have found interesting applications in WT simulations [TBC12, DCTB14].

The two cases where self-similar laws have been derived from the kinetic equation are 
numerically considered with the following assumptions: in both cases a rectangular plate is selected, transversely simply-supported with in-plane movable edges. More precisely we have for:

forced vibrations (case 1) : The plate is excited with a pointwise harmonic forcing for all the duration of the simulation. Both the excitation frequency and the forcing amplitude are kept constant. The excitation frequency is close to the 4th eigenfrequency of the plate. Damping is not taken into account.

free vibrations (case 2): The plate is forced pointwise impulsively at the start on a very short time interval, and then left free to vibrate. By doing so, a free turbulence regime settles down with a given, controlable amount of initial energy. Damping is not considered so that the dynamics is conservative.

Forced vibrations. A first case is considered with a $0.4 \times 0.6 \mathrm{~m}^{2}$ rectangular plate of thickness $h=1 \mathrm{~mm}$. The forcing, located at an arbitrary point, has excitation frequency at $75 \mathrm{~Hz}$, at a steady amplitude of $10 \mathrm{~N}$ (after an initial transient where the amplitude is increased from zero to the steady value). $102 \times 153$ grid points and a sampling rate of $400 \mathrm{kHz}$ are used. Fig. 1.3(a) shows the spectrogram of the displacement at an arbitrary output point: the absence of damping in addition to the steady forcing create a nonstationary turbulent cascade, with a front propagating to the high frequencies. A characteristic frequency defined as $f_{c}=\frac{\int_{0}^{\infty} P_{v}(f) f \mathrm{~d} f}{\int_{0}^{\infty} P_{v}(f) \mathrm{d} f}$ is introduced so as to quantitatively investigate the front, where $P_{v}(f)$ represents the velocity power spectrum in the frequency domain.
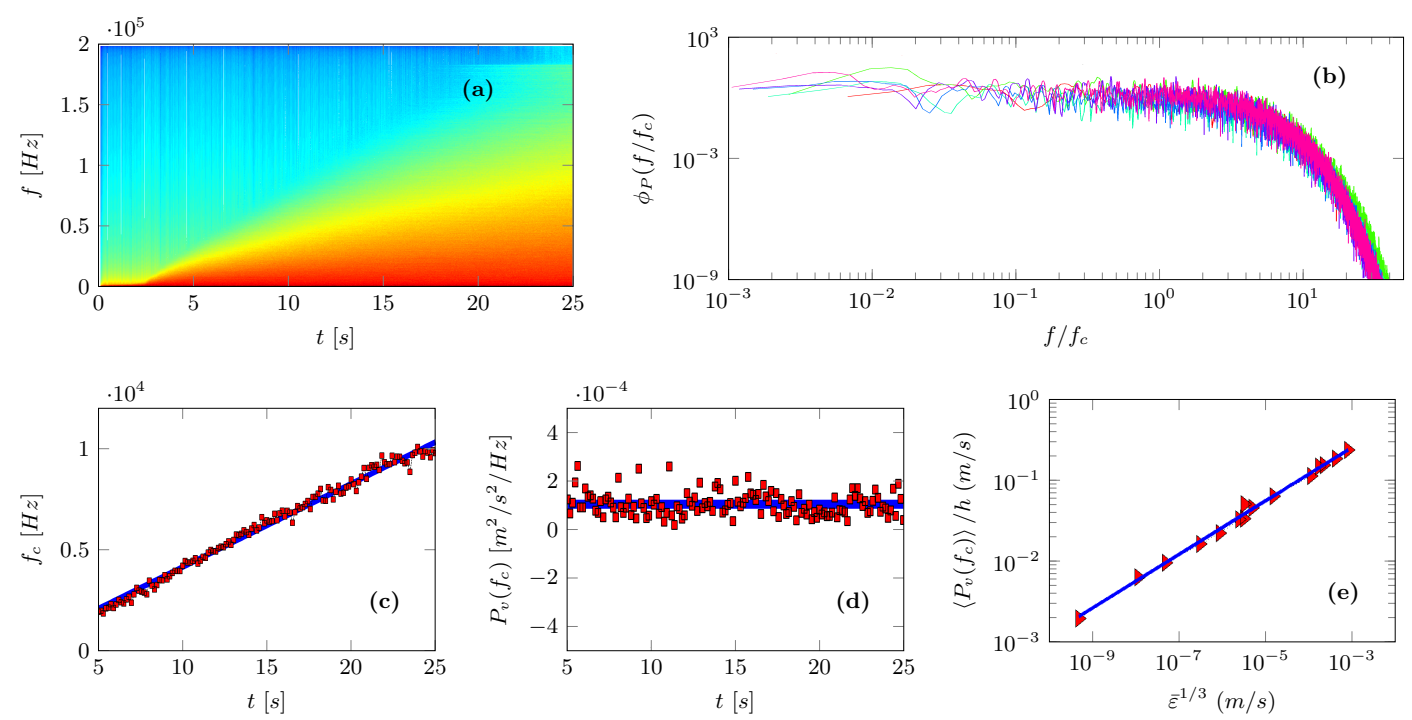

FIGURE 1.3 Nonstationary turbulence with steady forcing (case 1). (a): Spectrogram. (b): Normalised velocity power spectra. (c): Evolution of $f_{c}$ vs $t$. (d): Evolution of $P_{v}\left(f_{c}\right)$ vs $t$. (e): Scaling of spectral amplitude with injected power.

Fig. 1.3(c) and (d) show that $f_{c} \propto t$ and that the power spectrum at this characteristic frequency, $P_{v}\left(f_{c}\right)$, is constant. Plotting successive velocity spectra normalised by $f_{c}$ and $P_{v}\left(f_{c}\right)$ gives rise to Fig. 1.3(b), from which a self-similar dynamics is deduced. Therefore

$$
P_{v}(f)=P_{v}\left(f_{c}\right) \phi_{P}\left(\frac{f}{f_{c}}\right)=g_{1}\left(\frac{f}{t}\right) .
$$


This last equality, along with the fact that the energy in the system grows linearly over time, is in accordance with the theoretical prediction of nonstationary turbulence, Eq. (1.14). Other than agreeing formally with the theory, these simulations offer a visualisation of the shape of the self-similar function $g_{1}$, which is not given by the theory. Note that $g_{1}$ has the shape of $\phi_{P}$ used in Eq. (1.17) and shown in Fig. 1.3(b), where here the subscript $P$ refers to the periodic, constant amplitude forcing (case 1, forced vibrations). The nonstationary turbulence is in this case associated with a constant mean injected power $\bar{\epsilon}$ with increasing dispersion $\epsilon_{r m s}$ (see [DCTB14]). The cascade possesses various scaling laws. An important one, depicted in Fig. 1.3(e), relates the value of the mean injection $\bar{\epsilon}$ with the spectral amplitude $P_{v}\left(f_{c}\right)$. The figure, obtained from 15 simulations of plates with varying geometrical and forcing parameters, leads to the following expression: [DCTB14]:

$$
P_{v}\left(f_{c}\right) \propto h \bar{\epsilon}^{1 / 3} .
$$

This relationship shows that the power spectrum is proportional to the injected power at the power one third, in accordance with the fact that a four-waves interaction process is at hand [Naz11, DJR06, DCTB14].

Free turbulence. For this case where the plate is left free to vibrate, given an amount of energy as initial condition, a plate of sides $0.4 \times 0.6 \mathrm{~m}^{2}$ and thickness $h=0.1 \mathrm{~mm}$, is considered. The spectrogram of the displacement of an arbitrary output point is shown in Fig. 1.4(a). The behaviour of the front of the cascade $f_{c}(t)$ and the spectral amplitude $P_{v}\left(f_{c}\right)$ are shown in Figs. 1.4(c) and (d) with logarithmic scales, clearly exhibiting that $f_{c} \propto t^{1 / 3}$, and $P_{v}\left(f_{c}\right) \propto t^{-1 / 3}$. The normalised power spectra, in Fig. 1.4(b), show again a self-similar dynamics, so that $P_{v}(f)=P_{v}\left(f_{c}\right) \phi_{F}\left(\frac{f}{f_{c}}\right)=t^{-1 / 3} g_{2}\left(\frac{f}{t^{1 / 3}}\right)$ which again is in accordance with the theoretical predictions, Eq. (1.15). Again, thanks to the simulations one can appreciate the shape of the self-similar function $g_{2}$, represented through the function $\phi_{F}$ in Fig. 1.4(b), where the subscript $F$ stands now for free turbulence (case 2). Note that the shape of $\phi_{P}$ and $\phi_{F}$ are not exactly the same, when represented on the same figure one observes clearly that whereas the slope is almost perfectly flat in the free vibration case (function $\phi_{F}$, Fig. 1.4(b)), a small slope behaving as $f^{-0.2}$ is at hand for forced vibrations (function $\phi_{P}$, Fig. 1.3(b)). This highlights the fact that the pointwise forcing have a small effect on the slope of the spectra in the low-frequency range, as observed numerically as well as experimentally, see [DCTB14, MM11a].

\subsubsection{Effect of an imperfection}

Section 1.2.2 shows that the von Kármán equations for plates yield a 4-waves interaction term in the collision integral. Such equations apply exclusively to perfectly flat plates. In practice, real plates present local deformations that may alter its dynamical response [CTT09, TBC12]. When deformations are static, the von Kármán equations can be modified to account for them. Let $w_{0}$ denote a static deformation, then

$$
\begin{aligned}
& \rho h \ddot{w}+D \Delta \Delta w=\mathcal{L}\left(w+w_{0}, F\right), \\
& \Delta \Delta F=-\frac{E h}{2} \mathcal{L}\left(w, w+2 w_{0}\right) .
\end{aligned}
$$

The quadratic nonlinearity of the equations for imperfect plates translates in a 3 -waves process as a correction to the 4 -waves interactions of the perfectly flat plate. The question of whether or not such correction modifies the statistics of the turbulence has been investigated in [DCTB14], with a selected deformation of the form of a raised cosine at the centre of the plate. The amplitude of the imperfection has been chosen in a range from 1 to 10 

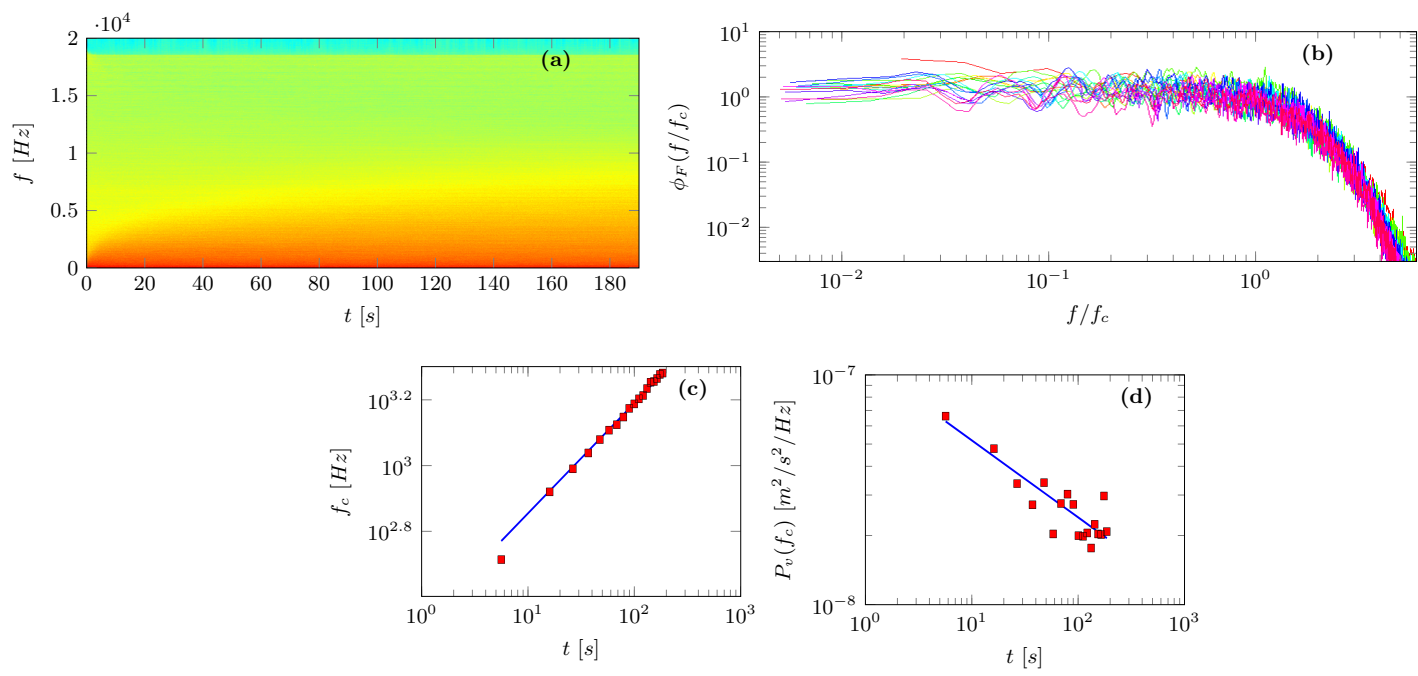

FIGURE 1.4 Nonstationary free turbulence (case 2). (a): Spectrogram. (b): Normalised velocity power spectra. (c): Evolution of $f_{c}$ vs $t$. (d): Evolution of $P_{v}\left(f_{c}\right)$ vs $t$.

times the thickness, and is shown in Fig. 1.5(a) (axis not in scale). Such choice is based on the consideration that real deformations in plates have the same order of magnitude, in general, at large wavelengths. Remarkably, the statistics of the turbulent regime is not affected by the presence of the imperfection. For obtaining this result, numerous simulations with different set-up have been computed in the framework of case 1, i.e. when the plate is constantly forced with a harmonic external excitation, and the different scaling laws have been found to be analog to those obtained for the perfect plate: the front propagates to high frequencies proportionnally to time and the amplitude of the power spectrum at the front is constant. Fig. 1.5(b) shows again a self-similar dynamics whose self-similar function does not differ from that of Fig. 1.3(b). The scaling law for the injected power is also unaffected: Fig. 1.5(c) shows that $P_{v}\left(f_{c}\right)=2.30 h \bar{\epsilon}^{1 / 3}$, statistically the same as Eq. (1.18) [DCTB14].
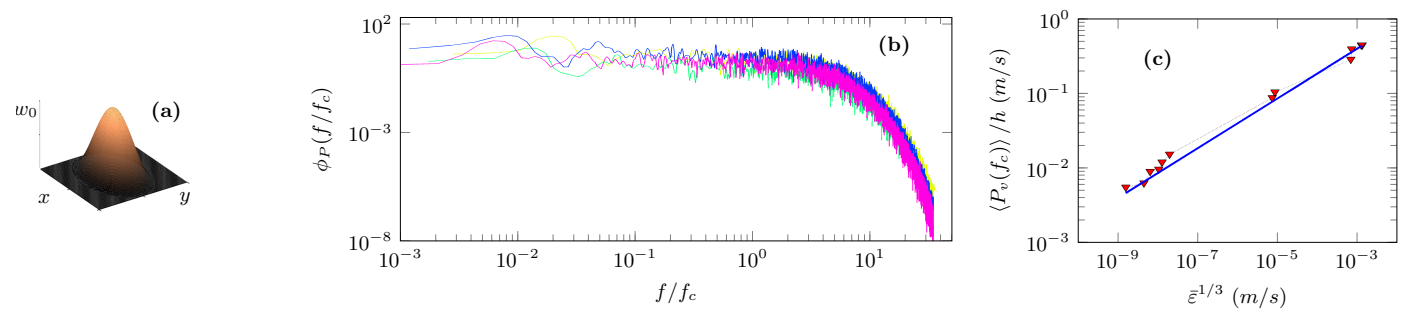

FIGURE 1.5 Nonstationary turbulence with steady forcing for imperfect plates. (a): Example of raised cosine imperfection $w_{0}$. (b): Normalised velocity power spectra. (c): Scaling of spectral amplitude with injected power.

These numerical results show that in the wave turbulence regime, the cubic nonlinearities dominate the quadratic ones, so that the effect of an imperfection should not affect the statistical properties of the dynamical solutions. 


\subsection{Real plates}

The two previous sections present the theoretical results and their numerical verification in two different cases, showing that when all the WT assumptions are fulfilled then the analytical predictions are numerically retrieved. We now turn to the case of real plates in order to see how the theoretical predictions confront with experiments.

\subsubsection{First experimental results}
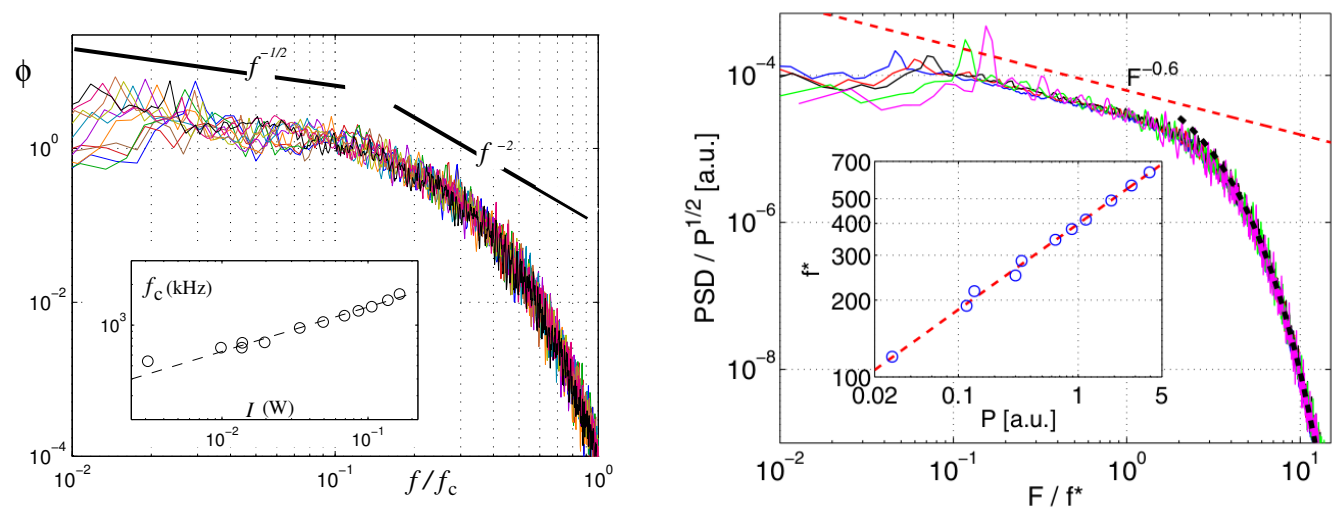

FIGURE 1.6 Rescaled power spectra of the transverse velocity obtained on two different experimental set-ups and reported in [BCOT08] (left) and [Mor08] (right). The power spectrum is rescaled by the square root of the injected power (denoted as $I$ on the left and $P$ on the right), while the frequency is rescaled by the cut-off frequency (denoted as $f_{c}$ on the left and $f^{\star}$ on the right). Both insert shows the dependence of the cut-off frequency with respect to the injected power.

The first experimental investigations on the WT in plates have been reported in 2008 on two different set-ups [BCOT08, Mor08]. In each case, a large (lateral dimensions $1 \times 2 \mathrm{~m}$ ) and thin (thickness around $0.4-0.5 \mathrm{~mm}$ ) plate, made of an homogeneous metallic material, has been selected. For exciting the plate in the turbulent regime, a shaker is used and creates a pointwise forcing. At this point, thanks to the measurement of the velocity, one is able to retrieve the experimental injected power. For the first measurements reported, the velocity of the transverse displacement was recorded with a laser vibrometer.

The two papers evidenced a discrepancy between the predicted power spectrum and the measurements. The turbulent behaviour is nonetheless confirmed, with the appearance of a large bandwidth and continuous spectrum having a cut-off frequency which is getting larger when one increases the injected power. Fig. 1.6 summarizes the obtained results by showing the rescaled power spectra of the transverse velocity. A clear self-similar behaviour is obtained in experiments and is highlighted by dividing the power spectrum by the square root of the injected power, while the frequency axis is made nondimensional by using the cut-off frequency. Both experiments show a scaling for the power spectrum as $P_{v}(\omega) \propto \varepsilon^{0.66} \omega^{-0.6}$, while the theory predicts $P_{v}(\omega) \propto \varepsilon^{0.33} \omega^{0}$. Considering the statistics of velocity increments, no intermittency has been measured [BCOT08]. Furthermore, using the correlations in a two-points measurement shows that the waves seem to be persistent [Mor08]. Hence the general framework of the WT was not called into question, but further investigation was needed to understand the origin of these discrepancies. 


\subsubsection{Weak nonlinearity and separation of timescales}

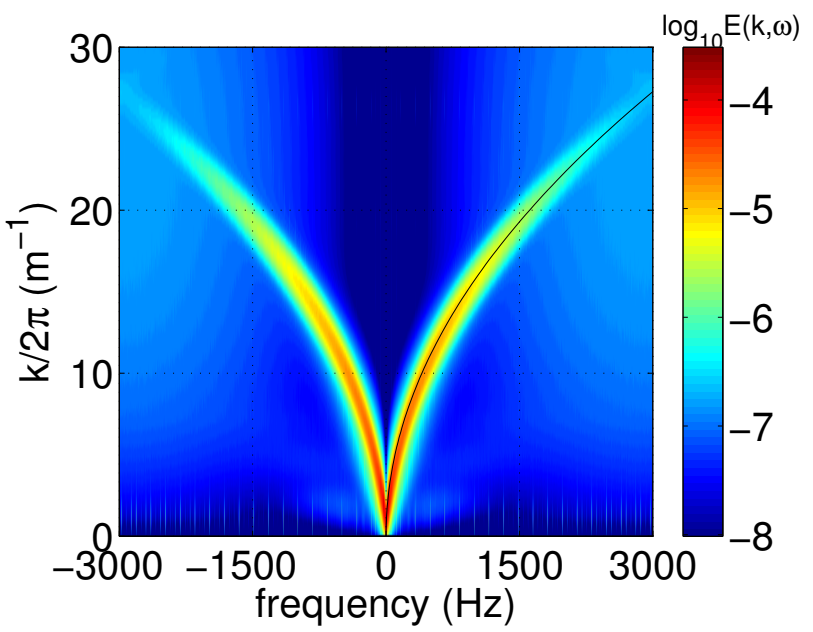

FIGURE 1.7 The angle integrated space-time spectrum $E(k, \omega)$ [Mor10]. The continuous line is the linear dispersion relation for the considered plate $\omega \propto k^{2}$.

Considering the previous observations that the spectrum of the vibration of the plate does not fulfill the predictions, the experimental investigations logically turns toward the verification of all the assumptions underlying the WT theory. In order to get a definitive answer to the very first assumption, stating that a wide spectrum of weakly nonlinear waves is at hand, dedicated experimental technique was developed in order to check whether a dispersion relation exists that is characteristic of propagating waves. The experimental difficulty of such a task is that a $2 \mathrm{D}$ space and time resolved measurement is required. This can be achieved by using a high-speed profilometry technique developed by Maurel and coworkers [MCPP09, CMPP]. The basic principle is to record with a high-speed camera a pattern projected on the plate. When the plate is deformed, the observed pattern is altered as well. For some patterns and for an adequate optical configuration, the images of the deformed pattern can be inverted to obtain the deformation of the plate. Thanks to this technique, movies of the deformation of the plate over a large area (typically over $1 \mathrm{~m}^{2}$ for a $1 \times 2 \mathrm{~m}^{2}$ steel plate) are obtained $\left[\mathrm{CPM}^{+} 09\right.$, Mor10]. The Fourier spectrum $E(\mathbf{k}, \omega)$ of the deformation can then be easily computed; it is found to be isotropic in $\mathbf{k}$. Hence we display on fig 1.7 a spectrum which has been summed over the directions of the wave vector $\mathbf{k}$. Two important conclusions can be drawn from this measurement: (i) the energy of the spectrum is concentrated on a dispersion relation, hence confirming that the motion is composed of waves (ii) the energy concentration lies in the very vicinity of the linear dispersion relation, evidencing that the waves are weakly nonlinear. The vibration of the plate is thus truly a turbulent state of weakly nonlinear waves.

One of the main hypothesis of the WT theory is the scale separation between the linear dynamics (oscillation of the waves) and the nonlinear dynamics (energy exchanges between waves). In fact, in the course of the derivation of the kinetic equation, the decomposition of the amplitudes as $A_{\mathbf{k}}=a_{\mathbf{k}}(t) \mathrm{e}^{i \omega_{k} t}$ is valid only if the time scale $T_{N L}$ of the wave modulation $a_{\mathbf{k}}(t)$ is large compared to the wave period $T=2 \pi / \omega_{k}$. For real plates an additional condition arises naturally: the dissipation time scale $T_{d}$ must be even longer so that nonlinear dynamics can develop. The final condition thus reads

$$
T \ll T_{N L} \ll T_{d} .
$$




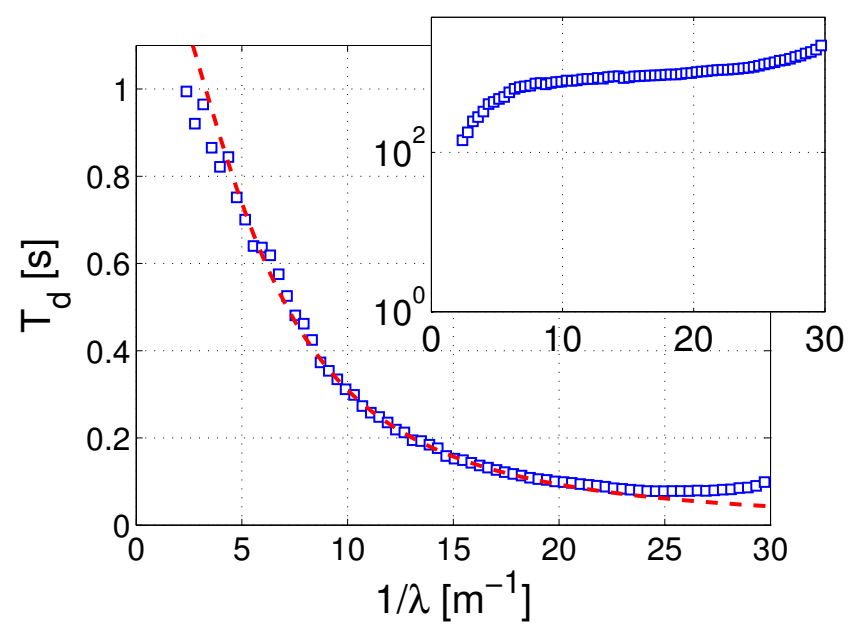

FIGURE 1.8 Squares: measured dissipative time $T_{d}$ versus $k / 2 \pi=1 / \lambda$. Dashed line: Lorentzian fit $T_{d}=\left(0.73+0.025(k / 2 \pi)^{2}\right)^{-1}$ used in the following. Insert: $T_{d} \omega$ versus $k / 2 \pi$ (semilog scale) [MM11a].

The dissipation time scale can be measured in various ways [MM11a, $\left.\mathrm{HCD}^{+} 13\right]$. One possibility is to run decay experiments from a fully turbulent state [MM11a]. The final decay state is exponential and corresponds to a linear dissipative decay of the wave amplitude. The dissipation rate can be extracted from this curve. Fig. 1.8 shows the dissipative time scales obtained with this method. The insert shows the product $T_{d} \omega$ that compares the dissipative time scale and the linear period of the wave. From this measurement two important conclusions can be drawn. First, the inequality $T \ll T_{d}$ is fulfilled as two orders of magnitude separates the two time scales for the wavelengths of interest. Second, the product $T_{d} \omega$ is almost constant with the wavenumber, showing that dissipation is weak but present at all frequencies.

In order to compare the nonlinear time scale with the linear ones, on has to extract the nonlinear dynamics from the experimental data, a challenging task since the measurement is operated only on a part of the full plate. Furthermore the real plate is finite so that reflections occur on the boundaries. The goal is to compute the time correlations of $a_{\mathbf{k}}(t)$ in order to extract the characteristic time scale of the nonlinear dynamics. The correlation coefficient can be reconstructed by performing a wave packet analysis [MM11a]. The deformation field is projected over Gaussian wavelets at various positions and for a given wavevector. This projection allows one to follow the propagation of the wave packets by correlating the wavelets coefficients at a given wavevector over various positions and times. Reflections on the boundaries can be used to follow the wave packets over longer times. When tracking the wave packets along their trajectory at the group velocity, the magnitude of the correlation of the wave packet magnitude is seen to decay with the propagation time. Two reasons account for this decay: (i) the dispersion of the wave packet, this effect can be easily corrected for Gaussian wave packets, (ii) the decay of the correlation due to the nonlinear energy exchanges among waves that destroy the coherence of the wave packet. Fig. 1.10 shows the dispersion-corrected decay of the temporal correlation of the wavelet coefficients for various positions. The decoherence of the wave packet is exponential so that the extraction of $T_{N L}$ is straightforward. Using this procedure, a time-space diagram of the decoherence of the wave packet is reconstructed on its trajectory (Fig. 1.10). When the forcing strength is increased, the decoherence of the wave packet is seen to be faster consistently with the expectation that the nonlinear dynamics is more developed when the forcing is stronger. 


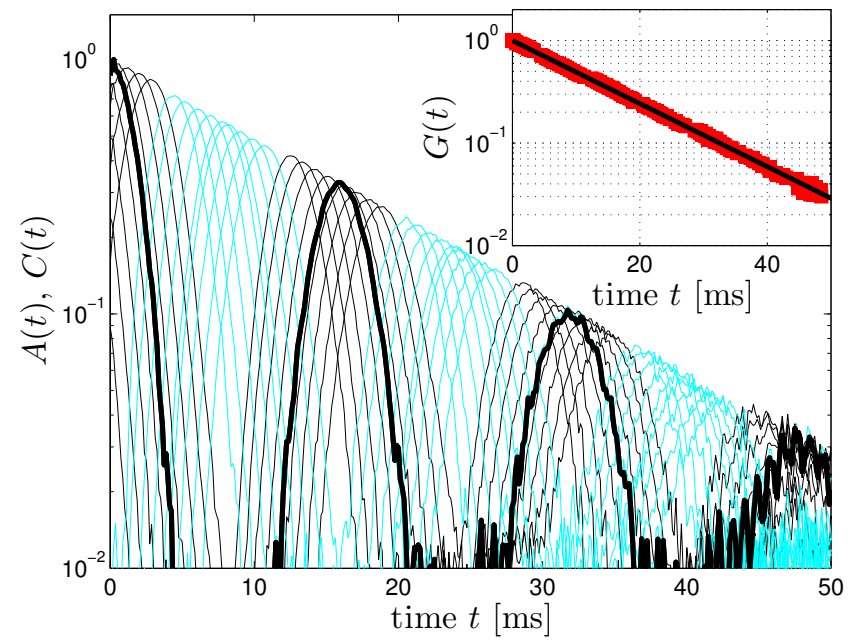

FIGURE 1.9 Decay of the coherence of a wave packet computed by correlation of wavelet coefficient at a given wavevector $\mathbf{k}$ for various positions. The abscissa axis is the propagation time from an initial position at the center of the plate. The dark blue curves are the correlations of wave packets with the same $\mathbf{k}$ (propagating in the same direction) for a set of positions on the trajectory of the wave packet. Hence, these curves correspond to wavepackets that have undergone an even number of reflections (or no reflection at all). The cyan curves are the correlation between counterpropagating wave packets that have an opposite wavevector $-\mathbf{k}$, as a result of an odd number of reflections. As time flows, the wavepacket propagates nearly balistically and bounces on the edges so that a succession of dark blue and cyan curves groupings is observed The envelop of the correlations curves is seen in the insert that shows that the overall decay of the coherence of the wave packet is exponential so that the extraction of the nonlinear time scale $T_{N L}$ is simply the characteristic time of the exponential decay [MM11a].

The extracted nonlinear timescale $T_{N L}$ is shown in Fig. 1.11 and compared to the dissipation time scale and the period of the wave. Thanks to the large separation between the two latter timescales, an intermediate range for the nonlinear timescale is possible. Indeed $T_{N L}$ lies inbetween these two scales. $T_{N L}$ is seen to vary very little with the wavenumber. It decays when the forcing strength is increased as mentioned above. Thus the double scale separation $T \ll T_{N L} \ll T_{d}$ is observed so that a true weak turbulence can develop in this system. Note a major difference with the canonical situation of the Weak Turbulence Theory: dissipation is weak but present at all scales and this leads naturally to a steepening of the wave spectrum as the energy flux is progressively decreased by dissipation. This point will be further addressed in section 1.4.4.

\subsubsection{Finite-size effects}

Another major hypothesis of the Weak Turbulence Theory is that the system is asymptotically large, so that the discreteness of the modes, which typical of finite size systems, is avoided. For small systems, a chaotic dynamics may develop in which the nonlinear time scales shows a distinct scaling [Kar94, LN10]. Indeed for small system the discreteness of the mode may restrict significantly the number of solutions of the resonance equations. Thus energy injected at the forcing scales must find a path to small scales through connected resonant quadruplets (or triplets for a three-waves interaction process). When the size of the system decreases, the topology of this clusters can become sparse, hence rendering the energy cascade difficult. 


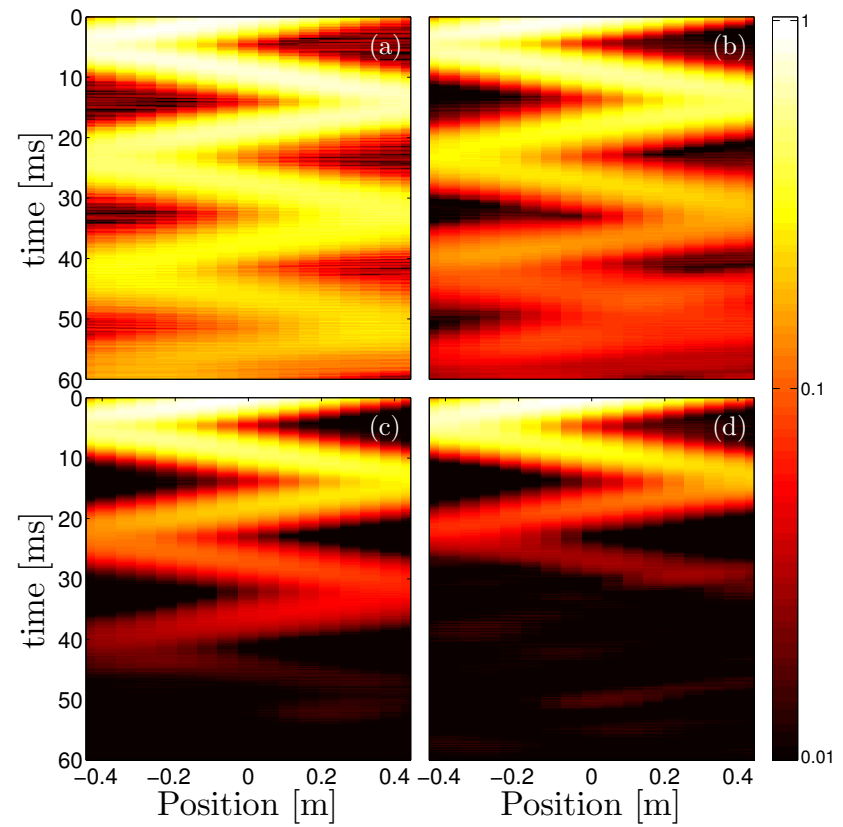

FIGURE 1.10 Space-time representation of the decoherence of the wave packets over their trajectory in the plate, including the bounces on the boundary. The initial position is at the center of the plate. Wavenumber $k / 2 \pi=13 \mathrm{~m}^{-1}$ for this specific example. Colors are log-coded and represent the correlation of the wave packets with its initial position. The forcing power is $P_{0}, 7 P_{0}, 27 P_{0}$ and $59 P_{0}$ (from (a) to (d)) [MM11a].

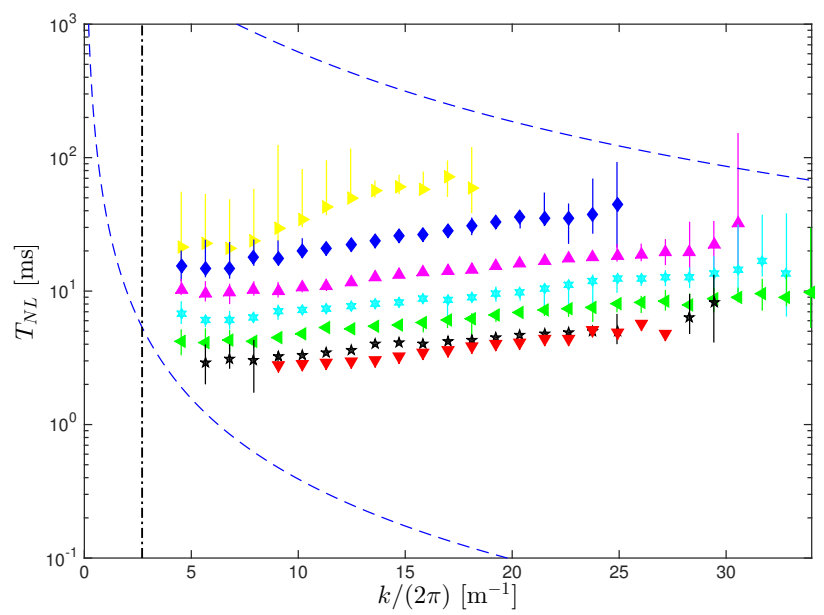

FIGURE 1.11 (Color online) Comparison of the nonlinear time scale with other relevant time scales. Symbols are experimental data for $T_{N L}$ at different values of the forcing intensity. Upper dashed line: twice the dissipative time $T_{d}$ measured by the decay of energy in the unforced case (see Fig. 1.8). Lower dashed line: $1 / \omega$. The forcing is operated at $30 \mathrm{~Hz}$ and is shown with the vertical dash-dotted line [MM11a].

When the size of the system is increased, the transition from a chaotic regime to a weak turbulence regime is actually an open question [NR11]. A qualitative description is the following: nonlinear effects induce a spectral widening of the modes. If the system is large 
enough, the spectral separation of the modes can become of the same order as this nonlinear widening. Consequently, the discreteness of the modes is destroyed by nonlinearity, and all frequency and wavenumber values are then possible, so that eventually a weak turbulence regime can develop.

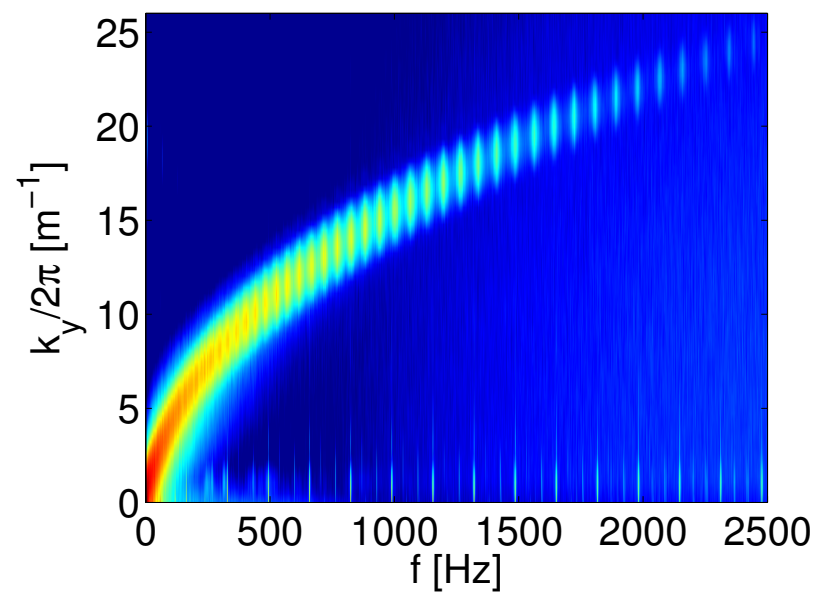

FIGURE 1.12 Cut of the space time spectrum $E(\mathbf{k}, \omega)$ at $k_{x}=0$. Discrete frequencies are clearly visible [Mor10].

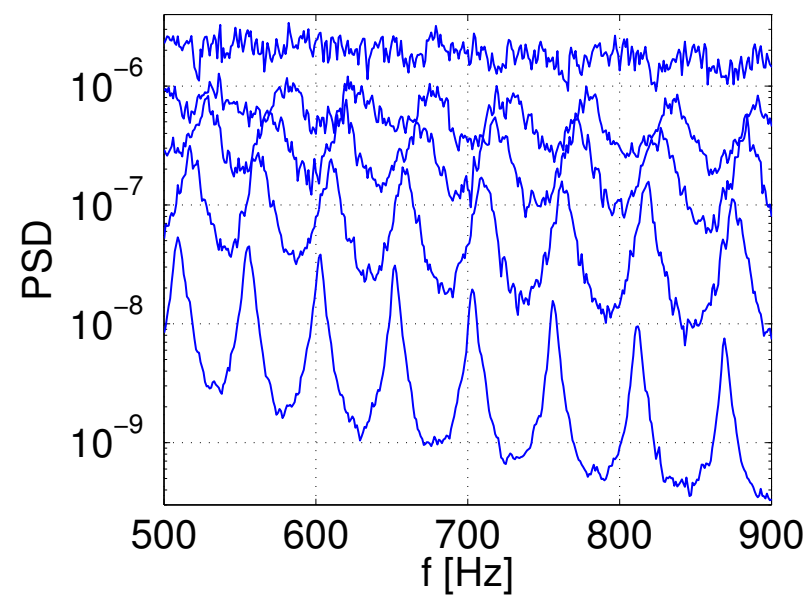

FIGURE 1.13 Evolution of the spectral energy on the dispersion relation in the plane $k_{x}=0$ for $P=1,4,9,16$ and 36 (from bottom to top). The curves have been shifted vertically by a factor 1.5 for clarity [Mor10].

Discrete modes can be observed for the experimental plate as can be seen in Fig. 1.12. The dispersion relation is continuous at low frequency but shows discrete peaks at the highest frequencies. This observation is related to the fact that the magnitude of the nonlinearity decreases when the frequency is increased. This can also be observed in Fig. 1.11: the ratio $T_{N L} / T$ increases with the frequency.

For a given frequency interval, Fig. 1.13 shows the evolution of the crest line of energy along the dispersion relation when the forcing intensity is increased. At very low forcing, 
the nonlinearity is very weak and the discrete peaks of the modes are clearly visible. When the forcing is increased the peaks enlarge (and their position is slightly shifted as well) due to the effect of nonlinearity. At the highest forcing intensity the spectrum is continuous. Note that the discrete character of the mode is visible for all forcing intensities but it appears at higher frequencies for larger forcing intensities. This feature can also be observed in numerical simulations of the plate. Thus a regime of weak turbulence develops at the lowest frequencies but evolves always into a regime of discrete turbulence at the highest frequencies that are observed in the spectrum. As a conclusion, the finite-size effect in the considered experimental set-up, though observable, should not be responsible for the important discrepancies found between theory and measurements.

\subsubsection{Effect of damping on the slope of the power spectra}

The last assumption to be tested is the transparency window or inertial range, i.e. the system shall display a large frequency range between the forcing and dissipative scales, where a conservative Hamiltonian dynamics can be assumed, so that the energy flux shall be conserved when cascading through scales. In solid plates, the dissipation has different physical origins, and in the set-up considered the main causes of losses are thermoelasticity, viscoelasticity, and dissipation at the boundaries. As already noted in the previous section, the damping, though small, is present at all scales, see also e.g. [CL01].

In $\left[\mathrm{HCD}^{+} 13\right]$, the effect of damping on the wave turbulence regime has been experimentally and numerically studied. The experimental plate is made of steel, the lateral dimensions are $2 \times 1 \mathrm{~m}^{2}$, and the thickness is $h=0.5 \mathrm{~mm}$. The plate is set into a turbulent regime with a sinusoidal forcing at frequency $f_{0}=30 \mathrm{~Hz}$. The natural damping of the plate is increased using different techniques based on paint and edge dampers. Four different configurations are studied and characterised by the measurements of the damping factors in the linear regime, denoted $\gamma$ and displayed as functions of the frequency in Fig. 1.14. Interestingly, despite the different attenuation sources, the damping factors exhibit always the same qualitative behaviour which can be characterised by a power law normalised so that

$$
\gamma(f)=\gamma^{*} f^{0.6}
$$

where $\gamma^{*}$ varies between 1 and 5 .

Fig. 1.15(a) displays the experimental power spectral densities of the normal velocity measured at similar injected powers for the four configurations. They all behave roughly as power laws in the cascade regime with frequency exponents that become clearly smaller as $\gamma^{*}$ increases. For the natural plate the exponent $(-0.5)$ is consistent with the previous results [BCOT08, Mor08], while for the most damped plate, the exponent is almost twice this value. In $\left[\mathrm{HCD}^{+} 13\right]$, numerical simulations of the von Kármán plate equations are also performed, using the same pseudo-spectral method than in [DJR06]. Within this framework, it is straightforward to inject energy at controlled scales and to mimic the measured experimental dissipation by using the fitted power laws displayed in Fig. 1.14. Fig. 1.15(b) shows the power spectral densities of the normal velocity obtained by numerical simulations for similar injected powers. The same behaviour as in the experiments is observed. The ideal case $\gamma^{*}=0$ with the almost flat Kolmogorov-Zakharov spectrum is drawn for comparison, arguing that the difference between the experimental and the theoretical values of the slope is mainly due to the existence of damping at all scales. Finally, in [MAM14], numerical simulations of the von Kármán equations are performed using three damping laws (displayed in Fig. 1.16(a)) going from the experimental dissipation to the one used in the theory of wave turbulence. Fig. 1.16(b) draws the numerical spectra associated to these three damping laws, showing that a transition from the experimental spectrum to the predicted KZ 


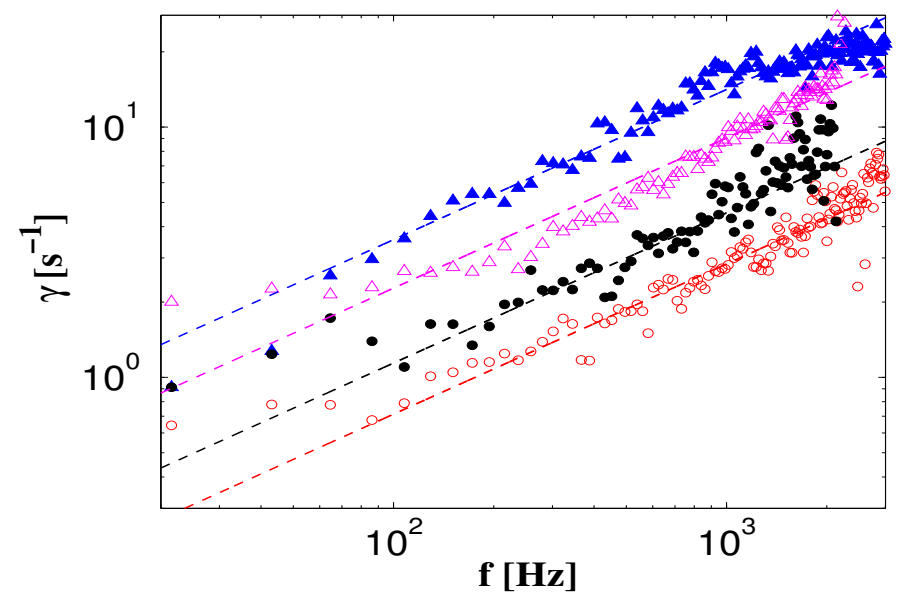

FIGURE 1.14 From $\left[\mathrm{HCD}^{+} 13\right]$. Evolution of the damping factor $\gamma$ as a function of the frequency. Empty red circles: natural plate. Filled black circles: one side painted. Empty magenta triangles: two sides painted. Filled blue triangles: two sides painted + edge dampers. Dashed lines: fitted power laws $\gamma \propto f^{0.6}$.
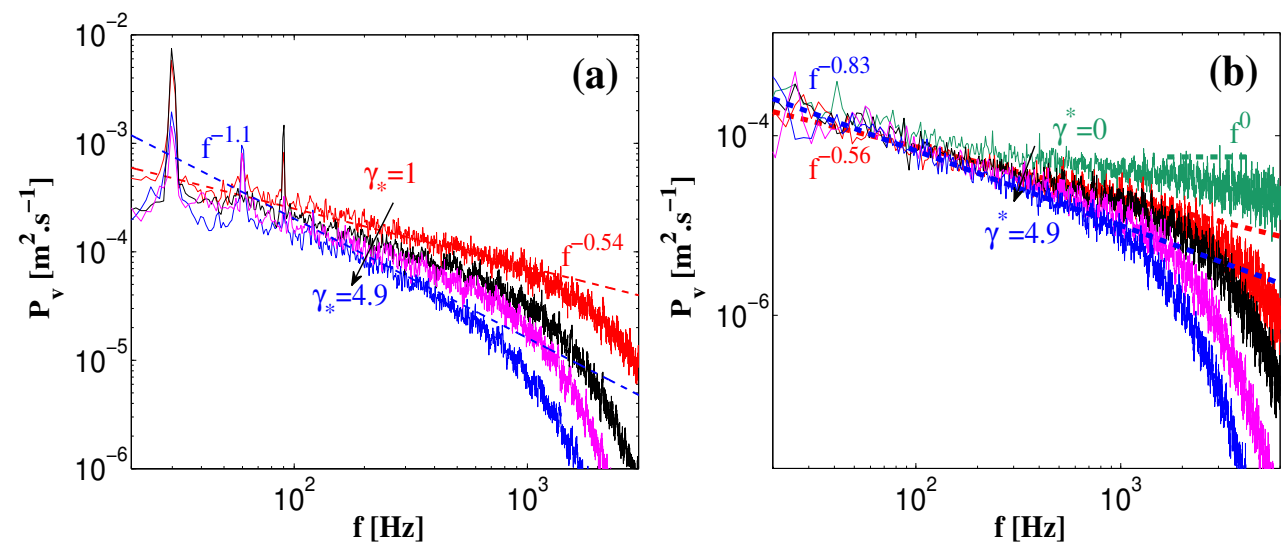

FIGURE 1.15 From $\left[\mathrm{HCD}^{+} 13\right]$. Power spectral density of the transverse velocity for the four configurations. Red dashed line + equation: smallest slope. Blue dashed line + equation: largest slope. (a) Experiments. Red: $\gamma^{*}=1, \varepsilon_{I}=0.56 \times 10^{-3} \mathrm{~m}^{3} \cdot \mathrm{s}^{-3}$. Black: $\gamma^{*}=1.6, \varepsilon_{I}=0.54 \times 10^{-3} \mathrm{~m}^{3} \cdot \mathrm{s}^{-3}$. Magenta: $\gamma^{*}=3.1, \varepsilon_{I}=0.52 \times 10^{-3} \mathrm{~m}^{3} \cdot \mathrm{s}^{-3}$. Blue: $\gamma^{*}=4.9, \varepsilon_{I}=0.48 \times 10^{-3} \mathrm{~m}^{3} \cdot \mathrm{s}^{-3}$. (b) Numerics. Green: $\gamma^{*}=0, \varepsilon_{I}=0.057 \mathrm{~m}^{3} \cdot \mathrm{s}^{-3}$. Other cases: $\varepsilon_{I}=0.024 \mathrm{~m}^{3} \cdot \mathrm{s}^{-3}$.

spectrum is observed when dissipation is decreased at large and medium scales.

These results clearly highlight that the slope of the turbulent power spectra in vibrating plates depends strongly on the damping, indicating that it must be retained as a pertinent feature to explain the difference between theory and experiments. Moreover, one can argue that because the dissipation is relevant at each scale, no inertial range (or transparency window) exists in these turbulent regimes so that the flux of energy is not constant over the cascade.

\subsubsection{Dependence on injected power}



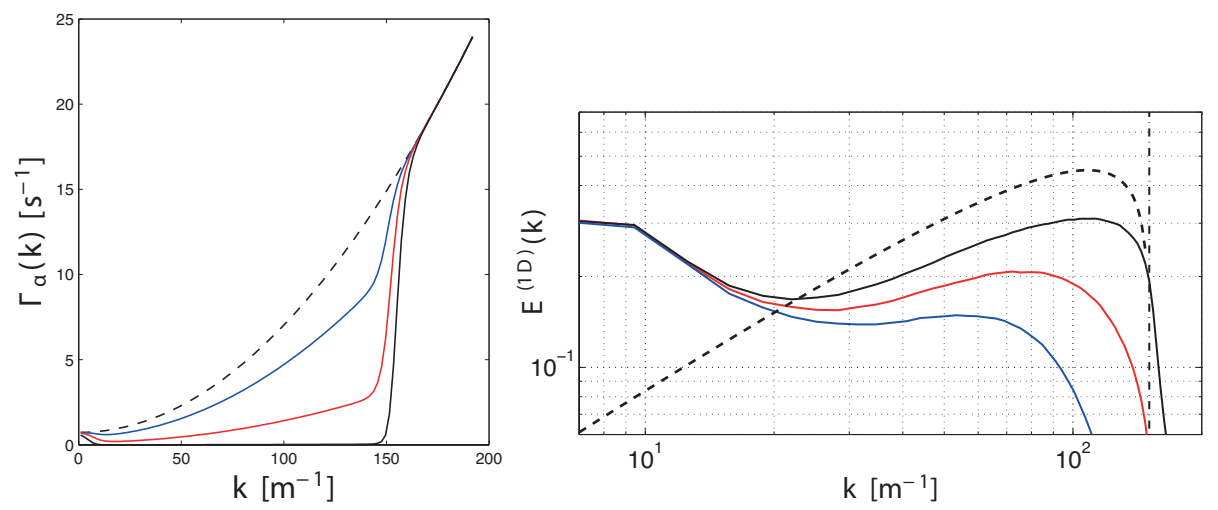

FIGURE 1.16 From [MAM14]. (Left) Dissipation $\Gamma_{\alpha}(k)$ (solid lines) compared to experimental dissipation $\gamma_{k}^{E X P}$ (black dashed line). (Right) Power spectral density $E^{(1 D)}(k)$. The black dashed line indicates the Kolmogorov-Zakharov spectrum.

A fundamental result of the WTT is the relationship between the number of resonant waves and the power exponent $\lambda$ of the energy flux in the power spectral density expression [ZLF92]. For three-waves interactions, the theory predicts $\lambda=\frac{1}{2}$ while four-waves interactions imply $\lambda=\frac{1}{3}$. For plates, the cubic nonlinearity of the restoring force implies four waves interactions, such that one would expect a $\frac{1}{3}$ power law on the energy flux. In the first reported experiments, see section 1.4.1 and [BCOT08, Mor08], the dependence of the power spectra on injected power was found to be near 0.6, i.e. very far from the expected value of $\frac{1}{3}$.

In order to obtain more quantitative results from the experiments, the energy budget of the cascade has been considered in [BCOT08, $\left.\mathrm{HCD}^{+} 13\right]$. The budget assumes that the cascade stops when the magnitude of the injected power has been completely dissipated by all the excited modes of the plate. Introducing $\varepsilon_{D}$ the dissipated power, one can write:

$$
\varepsilon_{D}=h \int_{0}^{\infty} \gamma(f) P_{v}(f) d f \simeq h \int_{0}^{f_{c}} \gamma(f) P_{v}(f) d f \propto \varepsilon_{I} .
$$

Considering a dissipation given by Eq. (1.21) and a spectrum of the form $P_{v}(f) \propto$ $\epsilon_{I}^{\lambda}\left(f / f_{c}\right)^{-\beta}$ with the exponent $\lambda$ and the slope $\beta$ unknown, the following expression for the cut-off frequency is obtained:

$$
\gamma_{*}^{1 / 1.6} f_{c} \propto \epsilon_{I}^{\frac{1-\lambda}{1.6}} .
$$

From the dependence of the cut-off frequency with respect to the injected power, one is able to retrieve the coefficient $\lambda$. It is found to vary from 0.36 to 0.57 in experiments and from 0.33 to 0.39 in numerics, depending on the damping coefficient. This result again shows that the presence of damping at all scales precludes for a direct comparison with the analytical predictions of the theory. A systematic deviation is observed, and the amount of discrepancy directly depends on the level of the damping in the system.

\subsection{Discussion}

Turbulence in a solid. Whereas the term turbulence generally refers to hydrodynamic turbulence and is somehow strongly related to the irregular motions of a fluid, the nonlinear 
interactions of a sea of coupled waves may also produce a regime of wave turbulence. In this context, the nonlinear vibrations of large and thin plates can be interpreted as an example of turbulence in a solid. The case of geometrically nonlinear vibrations of thin plates and shells, where the material's behaviour is considered as linear elastic so that the nonlinearity comes from the large-amplitude motions of the transverse deflection $w(\mathbf{x}, t)$, offers a correct framework to derive theoretical results thanks to the von Kármán model, as well as experimental results. Within this framework, the nonlinearity of the vibratory state is assessed by the ratio $w / h$ of the transverse displacement $w$ with respect to the thickness $h$ of the plate. When $w \ll h$, linear vibrations occurs. The von Kármán model has first been derived for vibration amplitudes that are of the order of the thickness. Though it may be valid for a larger range of amplitudes, numerical studies clearly assessing its validity limits in terms of amplitude, are still missing. On the experimental viewpoint, the range of amplitudes where the turbulence has been observed is: $h<w<50 h$.

Cascade of energy and turbulence. For the range of large-amplitude motions where $w>h$, the behaviour of the plate can be said as being truly turbulent. This fact is assessed by the broadband Fourier spectrum of the vibrations, the upper frequency of which depends on the energy level, evidencing a cascade of energy from the injection to a dissipative scale. This particular regime has musical applications in the understanding of the sound of gongs for example, where the higher frequency is reached a few milliseconds after the strike (the time the cascade to progress to higher frequencies) and the larger frequency is defined by the amplitude of the strike [DT15]. It has also been used in the past in theaters where large metallic plates were vigorously shaken to simulate the sound of thunder.

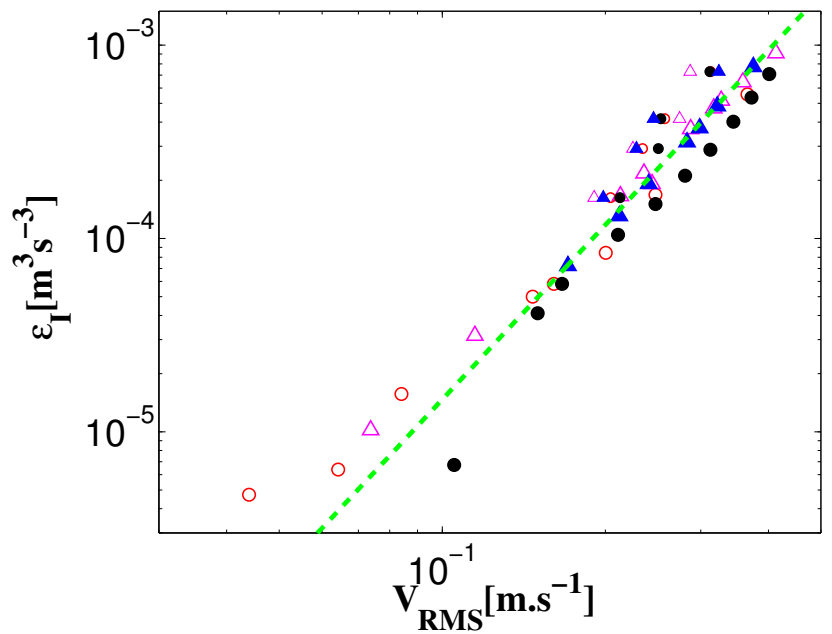

FIGURE 1.17 Injected power $\varepsilon_{I}$ versus root mean square value of the velocity at the injection point $V_{R M S}\left[\mathrm{HCD}^{+} 13\right]$. Large markers are used for experiments whereas small markers refers to numerical simulations. Variations of the damping as in section 1.4.4: red: $\gamma^{*}=1$, black: $\gamma^{*}=1.6$, magenta: $\gamma_{*}=3.1$, blue: $\gamma_{*}=4.9$, green: $\gamma^{*}=0$. Dashed green line $: \epsilon_{I} \propto V_{R M S}^{3}$.

The turbulent behaviour is also testified by the relation between the injected power and the injected velocity. Fig. 1.17 shows the injected power $\varepsilon_{I}$ as function of the root mean square value of the velocity at the injection point $V_{R M S}$, both in experiment and in numerical simulation, for the set-up described in section 1.4.4. For both cases, and for a damping coefficient $\gamma^{*}$ varying between 1 and 5 as in section 1.4.4, the dependence clearly exhibits 
the power-law: $\varepsilon_{I} \propto V_{R M S}^{3}$. This shows that the mechanism of power injection is inertial as observed in hydrodynamic turbulence when varying the viscosity $\left[\mathrm{CCD}^{+} 97\right]$.

Theory and experiments. The WT theory provides analytical results predicting the statistical repartition of energy through scales. Moreover, the self-similar solutions of the kinetic equation can be derived for predicting behaviour laws in the non-stationary case, though the theory does not yield the exact functional shape of the self-similar functions.

The discrepancy between the first experimental results reported in 2008 in [BCOT08, Mor08] and the analytical prediction derived in 2006 [DJR06] shall thus be attributed to the violation of an assumption used in the theoretical framework.

Checking the assumptions. Thanks to refined measurements, all hypothesis have been checked in a series of papers [BCOT08, Mor08, $\mathrm{CPM}^{+}$09, CBT08, Mor10, MM11a, CTB10, MM11b, HCD ${ }^{+} 13$, DCTB14, MAJM13, MAM14]. The persistence of waves and the correct separation of timescales has been fully documented in [Mor08, $\left.\mathrm{CPM}^{+} 09, \mathrm{MM} 11 \mathrm{~b}\right]$. As shown in section 1.4.3, finite-size effects are noticeable on the measurements but only at high frequencies where the vibration amplitude is getting exponentially small so that the nonlinearity is not sufficiently excited. In the frequency range of interest where the cascade develops, the energy spectrum is dense and sufficiently flat to assume that the finite-size effects are negligeable in the current experimental set-up.

Experimental set-up. Finally, typical effects induced by the experimental set-up could be invocated for explaining the observed discrepancies. Among these, the presence of imperfections in the static position are unavoidable with a real plate. However, numerical simulations shown in [DCTB14] clearly highlight that simple imperfections with large wavelength have no incidence on the dynamics of the power spectrum. As a matter of fact, at such vibration amplitudes, cubic nonlinearities dominate the quadratic ones so that the effect of the imperfection does not profundly modify the results. Another experimental bias is brought by the presence of a shaker, a device used for creating a pointwise excitation of the plate. The presence of this pointwise forcing has been shown to modify a little the slope of the spectrum around the forcing frequency. In experiment, this has been emphasized in [MM11a] in a non-stationary framework where the forcing was suddenly stopped once the plate in the WT regime. In the decay of turbulence, the slope of the spectrum was observed to flatten a little. Similar observations are shown in numerical nonstationary WT in [DCTB14], where the effect of the forcing is noticeable. However, this effect, though observable, remains small and is not the most salient feature for explaining the discrepancy between theory and measurements.

Effect of damping. The last assumption to validate is the transparency window. Thanks to experiments with varying damping ratios, and numerical simulations, recent studies undoubtedly shows that in real plates, all loss terms, though small, can not be neglected $\left[\mathrm{HCD}^{+} 13\right.$, MAM14]. As a consequence, the energy flux through scales is not constant, but slowly decreasing so that the amount of damping as function of the frequency defines both the slope of the power spectrum and the cut-off frequency.

\subsection{Conclusion}

Wave turbulence in nonlinearly vibrating plates have been thoroughly studied in this review chapter, which summarizes the most important results found since the publication of 
the theoretical results in 2006 [DJR06]. The picture of turbulence given in the theoretical framework is an idealization which is difficult or even impossible, to reproduce in a real experiment. A peculiar feature brought by the logarithmic correction in the $\mathrm{KZ}$ theoretical spectrum is that energy is assumed to completely disappear at the small scale introduced, $k_{\star}$, and which has no experimental counterpart. Thanks to measurements combined with numerical simulations, it has been clearly demonstrated that the presence of damping at all scales is the major effect for explaining the observed discrepancies. More precisely, the energy flux in the cascade is not constant, and this phenomenon precludes for a direct comparison with the theoretical results, as its effects is of prime importance on both the slope of the power spectrum and the dependence with injected power.

Further research in the direction of a better agreement between WT theory and experiments must then mandatory consider the effect of the damping in the WT calculations, as a first-order effect that needs to be taken into account in the kinetic equation. For that purpose, a phenomenological model may be used, see e.g. [HJTC16].

Other aspects may need further developments, either in numerical simulation or experimental verification. Recently, it has been shown numerically that a strongly nonlinear regime can be obtained for amplitudes of vibration $w$ that are larger than $100 h$, with $h$ the thickness [YT13, MAJM13, YT14]. This nonlinear regime is dominated by ridges connecting developable cones (D-cones), and scales linearly with the injected power. This observation supports the idea that the mechanisms responsible for spectral energy transfers differs strongly from the collisions between resonant waves stated by weak wave turbulence. Emergence of intermittency has also been reported in this context. Experimental measurements show that evidences of this regime may be observed when the gradient of the displacement is large enough [MAJM13]. However, for this range of amplitudes, the validity of the von Kármán model is questionable, so that further work is needed, both experimentally and numerically, in order to get insight into this strongly nonlinear regime.

Finally, the existence of an inverse cascade appears as a question witnessing new recent developments [DJR]. On the theoretical viewpoint, no stationary inverse cascade should exist in the framework of wave turbulence, in particular due to the non conservation of the wave action. However, when artificially neglecting the $3 \leftrightarrow 1$ interactions in the collision integral and thus conserving the number of waves in the interactions; an inverse cascade of wave action with constant flux can be derived [DJR]. Numerical simulations clearly exhibit this inverse cascade, thus questioning the nature of the involved interactions, as well the emergence of pattern and the possibility of self-organization. 


\section{References}

1. Z.P. Bazant and L. Cedolin. Stability of structures. World scientific, New-York, 1991.

2. A. Boudaoud, O. Cadot, B. Odille, and C. Touzé. Observation of wave turbulence in vibrating plates. Phys. Rev. Lett., 100:234504, 2008.

3. S. Bilbao. A family of conservative finite difference schemes for the dynamical von Kármán plate equations. Numerical Methods for Partial Differential Equations, 24(1):193-216, 2008.

4. S. Bilbao. Numerical Sound Synthesis. Wiley, 2008.

5. D. J. Benney and A.C. Newell. Sequential time closures of interacting random waves. J. Math. Phys., 363-393, 1967.

6. S. Bilbao, O. Thomas, C. Touzé, and M. Ducceschi. Conservative numerical methods for the full von Kármán plate equations. Num. Meth. for Partial Diff. Eq., submitted, 2014.

7. O. Cadot, A. Boudaoud, and C. Touzé. Statistics of power injection in a plate set into chaotic vibration. Eur. Phys. J. B, 66:399-407, 2008.

8. O. Cadot, Y. Couder, A. Daerr, S. Douady, and A. Tsinober. Energy injection in closed turbulent flows: Stirring through boundary layers versus inertial stirring. Phys. Rev. E, 56(1):427-433, 1997.

9. H. N. Chu and G. Herrmann. Influence of large amplitudes on free flexural vibrations of rectangular elastic plates. Journal of Applied Mechanics, 23:532-540, 1956.

10. P.G. Ciarlet. A justification of the von Kármán equations. Arch. Rat. Mech. analysis, 73:349-389, 1980.

11. A. Chaigne and C. Lambourg. Time-domain simulation of damped impacted plates. I: Theory and experiments. The Journal of the Acoustical Society of America, 109:1422, 2001.

12. P. J. Cobelli, A. Maurel, V. Pagneux, and P. Petitjeans. Global measurement of water waves by Fourier transform profilometry. Exp. Fluids.

13. C. Connaughton, A. Newell, and Y Pomeau. Non-stationary spectra of local wave turbulence. Physica D, 184:64-85, 2003.

14. P. Cobelli, P. Petitjeans, A. Maurel, V. Pagneux, and N. Mordant. Space-time resolved wave turbulence in a vibrating plate. Phys. Rev. Lett., 103(20):204301, 2009.

15. O. Cadot, C. Touzé, and A. Boudaoud. Linear versus nonlinear response of a forced wave turbulence system. Physical Review E, 82:046211, 2010.

16. C. Camier, C. Touzé, and O. Thomas. Non-linear vibrations of imperfect free-edge circular plates and shells. European Journal of Mechanics - A/Solids, 28:500 $515,2009$.

17. M. Ducceschi, O. Cadot, C. Touzé, and S. Bilbao. Dynamics of the wave turbulence spectrum in vibrating plates: A numerical investigation using a conservative finite difference scheme. Physica D, 280-281:73-85, 2014.

18. G. Düring, C. Josserand, and S. Rica. Self-similar formation of an inverse cascade in vibrating elastic plates. Physical Review E, submitted.

19. G. Düring, C. Josserand, and S. Rica. Weak turbulence for a vibrating plate: Can one hear a Kolmogorov spectrum? Phys. Rev. Lett., 97:025503, 2006.

20. S. Dyachenko, A. C. Newell, A. Pushkarev, and V. E. Zakharov. Optical turbulence: weak turbulence, condensates and collapsing filaments in the nonlinear Schrödinger equation. Physica D, 57:96-160, 1992.

21. M. Ducceschi and C. Touzé. Modal approach for nonlinear vibrations of damped impacted plates: application to sound synthesis of gongs and cymbals. Journal of Sound and Vibration, Accepted for publication, 2015.

22. G. Düring. Non-equilibrium dynamics of nonlinear wave systems : Turbulent regime, 
breakdown and wave condensation. PhD thesis, Université Pierre et Marie Curie, Paris 6, 2010.

23. A. Föppl. Vorlesungen über technische Mechanik, volume 5. Druck und Verlag von B. G. Teubner, 1907.

24. U. Frisch. Turbulence. The Legacy of A.N. Kolmogorov. Cambridge University Press, Cambridge, 1995.

25. G.E. Falkovich and A.V. Shafarenko. Nonstationary wave turbulence. Journal of Nonlinear Science, 1:457-480, 1991.

26. T. Humbert, O. Cadot, G. Düring, C. Josserand, S. Rica, and C. Touzé. Wave turbulence in vibrating plates : the effect of damping. EPL, 102:30002, 2013.

27. T. Humbert, C. Josserand, C. Touzé, and O. Cadot. Phenomenological model for predicting stationary and non-stationary spectra of wave turbulence in vibrating plates. Physica D: Nonlinear Phenomena, 316:34 - 42, 2016.

28. E. Kartashova. Weakly nonlinear theory of finite-size effects in resonators. Phys. Rev. Lett., 72(13):2013-2016, Jan 1994.

29. A. N. Kolmogorov. The local structure of turbulence in incompressible viscous fluid for very large Reynolds number. Dokl. Akad. Nauk. SSSR, 30:9-13, 1941.

30. A. N. Kolmogorov. On degeneration (decay) of isotropic turbulence in an incompressible viscous liquid. Dokl. Akad. Nauk. SSSR, 31:538-540, 1941.

31. R. M. Kirby and Z. Yosibash. Solution of von Kármán dynamic non-linear plate equations using a pseudo-spectral method. Computer Methods in Applied Mechanics and Engineering, 193:575-599, 2004.

32. L. D. Landau and E. M. Lifshitz. Theory of elasticity. Pergamon Press, 1959.

33. V. S L'vov and S. Nazarenko. Discrete and mesoscopic regimes of finite-size wave turbulence. Phys. Rev. E, 82(5):056322, 2010.

34. Y. L'vov, S. V. Nazarenko, and R. West. Wave turbulence in Bose-Einstein condensates. Physica D, 184:333-351, 2003.

35. B. Miquel, A. Alexakis, C. Josserand, and N. Mordant. Transition from wave turbulence to dynamical crumpling in vibrated elastic plates. Phys. Rev. Lett., 111(5):054302, 2013.

36. B. Miquel, A. Alexakis, and N. Mordant. The role of dissipation in flexural wave turbulence: from experimental spectrum to Kolmogorov-Zakharov spectrum. Physical Review E, 89(6):062925, 2014.

37. A. Maurel, P. Cobelli, V. Pagneux, and P. Petitjeans. Experimental and theoretical inspection of the phase-to-height relation in Fourier transform profilometry. Appl. Optics, 48:380-392, 2009.

38. O. Millet, A. Hamdouni, and A. Cimetière. Justification du modèle bidimensionnel nonlinéaire de plaque par développement asymptotique des équations d'équilibre. $C$. R. Académie des Sciences IIb, 324:349-354, 1997.

39. B. Miquel and N. Mordant. Non stationary wave turbulence in an elastic plate. Phys. Rev. Lett., 107(3):034501, 2011.

40. B. Miquel and N. Mordant. Nonlinear dynamics of flexural wave turbulence. Phys. Rev. E, 84(6):066607, 2011.

41. N. Mordant. Are there waves in elastic wave turbulence? Phys. Rev. Lett., 100(23):234505, 2008.

42. N. Mordant. Fourier analysis of wave turbulence in a thin elastic plate. Eur. Phys. J. $B, 76: 537-545,2010$.

43. S. L. Musher, A. M. Rubenchik, and V. E. Zhakarov. Weak Langmuir turbulence. Phys. Rep., 252:177-274, 1995.

44. S. Nazarenko. Wave Turbulence. Springer-Verlag, Berlin Heidelberg, 2011.

45. A. H. Nayfeh and D. T. Mook. Nonlinear oscillations. John Wiley \& sons, New-York, 
1979.

46. A. C. Newell, S. Nazarenko, and L. Biven. Wave turbulence and intermittency. Physica D, 152-153, 2001.

47. S. V. Nazarenko, A. C. Newell, and S. Galtier. Non-local mhd turbulence. Physica D, 152-153:646-652, 2001.

48. A. C. Newell and B. Rumpf. Wave turbulence. Annu. Rev. Fluid Mech., 43:59-78, 2011.

49. M. Onorato, A. R. Osborne, M. Serio, D. Resio, A. Pushkarev, V. E. Zakharov, and C. Brandini. Freely decaying weak turbulence for sea surface gravity waves. Phys. Rev. Lett., 89:144501, 2002.

50. A. N. Pushkarev and V. E. Zakharov. Turbulence of capillary waves. Phys. Rev. Lett., 76:3320-3323, 1996.

51. S. Sridhar, D. T. Mook, and A. H. Nayfeh. Non-linear resonances in the forced responses of plates, part I: symmetric responses of circular plates. Journal of Sound and Vibration, 41(3):359-373, 1975.

52. O. Thomas and S. Bilbao. Geometrically nonlinear flexural vibrations of plates: Inplane boundary conditions and some symmetry properties. Journal of Sound and Vibration, 315(3):569-590, 2008.

53. C. Touzé, S. Bilbao, and O. Cadot. Transition scenario to turbulence in thin vibrating plates. Journal of Sound and Vibration, 331(2):412-433, 2012.

54. C. Touzé, O. Thomas, and A. Chaigne. Asymmetric non-linear forced vibrations of freeedge circular plates, part I: theory. Journal of Sound and Vibration, 258(4):649676, 2002.

55. C. Touzé, M. Vidrascu, and D. Chapelle. Calcul direct de la raideur non linéaire géométrique pour la réduction de modèles de coques en éléments finis. In Proceedings of CSMA 2013, Colloque national en calcul de structures, Giens, May 2013.

56. C. Touzé, M. Vidrascu, and D. Chapelle. Direct finite element computation of nonlinear modal coupling coefficients for reduced-order shell models. Computational Mechanics, 54(2):567-580, 2014.

57. T. von Kármán. Festigkeitsprobleme im maschinenbau. Encyklopädie der Mathematischen Wissenschaften, 4(4):311-385, 1910.

58. Z. Yosibash and R. M. Kirby. Dynamic response of various von Kármán non-linear plate models and their 3-d counterparts. International Journal of Solids and Structures, 42:2517-2531, 2005.

59. N. Yokoyama and M. Takaoka. Weak and strong wave turbulence spectra for elastic thin plate. Phys. Rev. Lett., 110(10):105501, 2013.

60. N. Yokoyama and M. Takaoka. Identification of a separation wave number between weak and strong turbulence spectra for a vibrating plate. Physical Review E, 89:012909, 2014.

61. V. E. Zakharov and N. N. Filonenko. Energy spectrum for stochastic oscillations of surface of a liquid. Sov. Phys. Dokl., 11:881-884, 1967.

62. V. E. Zakharov and N. N. Filonenko. Weak turbulence of capillary waves. J. Appl. Mech. Tech. Phys., 8:37-42, 1967.

63. V. E. Zakharov, V. S. L'vov, and G. Falkovich. Kolmogorov Spectra of Turbulence 1: Wave Turbulence. Series in Nonlinear Dynamics, Springer, 1992. 